BUTP-94/22

\title{
Group Quantization of Parametrized Systems II. Pasting Hilbert spaces
}

\author{
P. Hájičcek, A Higuchi and J. Tolar円 \\ Institute for Theoretical Physics \\ University of Bern \\ Sidlerstrasse 5, CH-3012 Bern, Switzerland
}

December 1994

\begin{abstract}
The method of group quantization described in the preceeding paper I is extended so that it becomes applicable to some parametrized systems that do not admit a global transversal surface. A simple completely solvable toy system is studied that admits a pair of maximal transversal surfaces intersecting all orbits. The corresponding two quantum mechanics are constructed. The similarity of the canonical group actions in the classical phase spaces on the one hand and in the quantum Hilbert spaces on the other hand suggests how the two Hilbert spaces are to be pasted together. The resulting quantum theory is checked to be equivalent to that constructed directly by means of Dirac's operator constraint method. The complete system of partial Hamiltonians for any of the two transversal surfaces is chosen and the quantum Schrödinger or Heisenberg pictures of time evolution are constructed.
\end{abstract}

\footnotetext{
${ }^{1}$ Present address: Department of Physics and Doppler Institute, Faculty of Nuclear Sciences and Physical Engineering, Czech Technical University, Břehová 7, CZ-115 19 Prague 1, Czech Republic (e-mail: tolar@br.fjfi.cvut.cz).
} 


\section{Introduction}

Quantization of first-class parametrized systems, like general relativity or string theory, is an outstanding problem in theoretical physics. In ref. [1], which will be referred to as I in this paper, a theory has been worked out for parametrized systems of finite dimensions in which a crucial role is played by gauge invariant objects like algebras of perennials or groups of symmetries; the theory combines ideas on the forms of relativistic dynamics [2] and on algebraic [3] and group [4] quantization. The problem of time, which is characteristic of the quantization of parametrized systems, emerges in the form of "frozen dynamics problem" [5]. The proposal in I of how this problem can be solved goes back to Dirac's notions of transversal surface and Hamiltonian [2]. A transversal surface represents a particular gauge, and a system of Hamiltonians defines a family of time levels in classical solutions. It is shown in refs. [1] and [6] that different choices of transversal surfaces and Hamiltonians lead to the same quantum theories in the sense that the predictions for one and the same measurement is independent of these choices. In other words, the so-called "multiple choice problem" [5], which is typical of quantization methods that have to specify a choice of time foliation, does not afflict our theory. In order to obtain these results, however, the existence of a so-called global transversal surface was assumed. Thus, we still have to confront the "global time" [5] and "Gribov problems": There are many parametrized systems which do not admit a global transversal surface.

As yet we are not able to give a general solution to this difficult problem; the present paper is just a small exercise: we limit ourselves to a very simple and completely solvable system for which there is no global transversal surface and we describe some method of solution to the problem at least for this system. The idea is, roughly speaking, taken over from the theory of differentiable manifolds: if there is no global coordinate system, then there will be coordinate patches; one can calculate within each patch and transform the results between the patches where they overlap. Similarly, one can choose a system of transversal surfaces which together intersect all gauge orbits and which are maximal in the sense of paper I. Each of these surfaces defines a reduced system of its own, for which quantum theories can be constructed. Then the quantum theories corresponding to different transversal surfaces are "pasted together" in a self-consistent way so that a single quantum theory results. In particular, the Hilbert spaces are pasted together so that again a Hilbert space is obtained (and not, say, the more general Hilbert manifold). The pasting method is based on the property of the groups of symmetries that they "remember" the global structure of the transversal surfaces.

The plan of the paper is as follows. In Sec. 2, we define the classical model and study its constraint surface, the topology of the orbits in the constraint surface that is relevant to the nonexistence of a global transversal surface, and list some peren- 
nials. In Sec. 3, a particular class of symmetries of the system is considered, namely the transformations which act linearly in the extended phase space. All continuous linear symmetries from the group $\mathrm{SO}_{2}(2,1)$ (the subscript " 2 " denotes the double covering); in addition, two discrete symmetries, $\sigma$ and $\tau$ are found, which may be related to the parity and time reversal in the 3-dimensional "Minkowski" space of the fundamental representation of $S O(2,1)$. Then, we choose the group $O_{2}^{+}(2,1)$ (this is the double covering of the orthochronous Lorentz group in three dimensions; it includes the parity $\sigma$ ) as the first-class canonical group of the system (cf. I). The action of $O_{2}^{+}(2,1)$ on the constraint hypersurface is then explicitly calculated. In Sec. 4 , we choose a pair $\Gamma_{1}, \Gamma_{2}$ of maximal transversal surfaces, find their symplectic structure, determine their overlapping and project the action of the group $O_{2}^{+}(2,1)$ to them. It turns out that due to the inclusion of $\sigma$, the group acts transitively on both transversal surfaces, and has a subgroup, namely $S_{2}(2,1)$, which acts transitively just within their overlapping subsets. In Sec. 5, we choose a representation of the symmetry group. The method of constructing the representation is just the canonical quantization of the reduced systems associated with each transversal surface. In this way, one obtains a reducible representation which decomposes into two irreducible ones. Only one of these, however, is faithful and so the choice, at least between these two obtained by canonical quantization, is easy. Moreover, the chosen representation of $\mathrm{O}_{2}^{+}(2,1)$ decomposes into two irreducible representations of its subgroup $\mathrm{SO}_{2}(2,1)$. Then, with each (classical) phase space $\Gamma_{i}, i=1,2$, we associate a (quantum) Hilbert space $\mathcal{H}_{i}$ carrying the above representation of $\mathrm{O}_{2}^{+}(2,1)$. $\mathcal{H}_{i}$ has two subspaces, $\mathcal{H}_{i}^{ \pm}$, each of which carries an irreducible representation of $\mathrm{SO}_{2}(2,1)$. This is analogous to $\Gamma_{i}$ having two open submanifolds, $\Gamma_{i}^{ \pm}$, in which $\Gamma_{1}$ and $\Gamma_{2}$ overlap, and within which $S_{2}(2,1)$ acts transitively. This analogy between the action of these groups in the classical and quantum theory suggests the construction of quantum mechanics that is performed in Sec. 6. There, we identify the subspace $\mathcal{H}_{1}^{+}$with $\mathcal{H}_{2}^{+}$and $\mathcal{H}_{1}^{-}$with $\mathcal{H}_{2}^{-}$using the unitary transformations

$$
U_{ \pm}: \mathcal{H}_{1}^{ \pm} \rightarrow \mathcal{H}_{2}^{ \pm}
$$

that map the corresponding equivalent representations of $\mathrm{SO}_{2}(2,1)$ into each other. These "pasting maps" $U_{ \pm}$are determined up to phase factors (Schur lemma!). By the requirement that the parity acts on the Hilbert space obtained by the identification, the relative phase factor is fixed. Sec. 7 contains a proof that the pasting construction yields a quantum theory that is equivalent to the quantum theory of the same system obtained by the Wheeler-DeWitt-equation method - a method which dispenses of transversal surfaces. Thus, certain "convergence" of methods is demonstrated. Finally, in Sec. 8, a system of Hamiltonians is chosen, the corresponding family of time levels is shown to be complete and the Schrödinger or Heisenberg picture of time evolution is described in detail. 


\section{The model}

In this section, we describe the chosen simple model. The (extended) phase space $\Gamma$ is $\left(\mathbb{R}^{4}, \Omega\right)$. In global Cartesian coordinates $q_{1}, q_{2}, p_{1}, p_{2}$ the symplectic form is $\Omega=d p_{1} \wedge d q_{1}+d p_{2} \wedge d q_{2}$. The definition of our parametrized system is finished by specifying the constraint:

$$
C=\frac{1}{2}\left(p_{1}^{2}-p_{2}^{2}-q_{1}^{2}+q_{2}^{2}\right)=0 .
$$

The model has a purely quadratic constraint $C$. Thus, the equations of motion are linear, with constant coefficients, and the system is completely solvable. Still, the space of $C$-orbits (classical solutions) is non-Hausdorff and there are three topologically different kinds of orbits: free orbits, which definitely leave any compact subset of $\Gamma$ in both directions, orbits with a limit point in the future or the past, and one critical point. As proposed in I, we remove the critical point from the phase space. Thus, from now on $\Gamma$ stands for $\mathbb{R} \backslash\{(0,0,0,0)\}$.

\subsection{Constraint hypersurface}

Let $\tilde{\Gamma}$ denote the constraint hypersurface defined by eq. (四). Introduce coordinates $r, \alpha, \beta$ on $\tilde{\Gamma}$ by

$$
\begin{aligned}
& q_{1}=-r \cos \beta, \quad p_{1}=r \cos \alpha, \\
& q_{2}=r \sin \alpha, \quad p_{2}=r \sin \beta .
\end{aligned}
$$

For $r>0, \alpha, \beta \in[0,2 \pi)$, we obtain all points of $\tilde{\Gamma}$. The topology is

$$
\tilde{\Gamma}=S^{1} \times S^{1} \times \mathbb{R}_{+} .
$$

To visualize the orbits and find the symmetries, a canonical transformation to the following set of canonical coordinates is useful:

$$
\begin{array}{ll}
Q_{1}=\frac{1}{\sqrt{2}}\left(q_{1}+p_{1}\right), & P_{1}=\frac{1}{\sqrt{2}}\left(-q_{1}+p_{1}\right), \\
Q_{2}=\frac{1}{\sqrt{2}}\left(q_{2}-p_{2}\right), & P_{2}=\frac{1}{\sqrt{2}}\left(q_{2}+p_{2}\right) .
\end{array}
$$

In the new coordinates, the constraint reads

$$
C=Q_{1} P_{1}+Q_{2} P_{2}
$$

Any point of $\Gamma$ is determined by two vectors

$$
Q=\left(Q_{1}, Q_{2}\right), \quad P=\left(P_{1}, P_{2}\right)
$$


which can be pictured as vectors in the $Q$ - and $P$-planes, respectively. The constraint $C=0$ just means that they are 'orthogonal'. This leads us to the third coordinate system on $\tilde{\Gamma}$ : we define

$$
\begin{aligned}
m(\psi) & =\left(-\sin \frac{\psi}{2}, \cos \frac{\psi}{2}\right), \\
n(\psi) & =\left(\cos \frac{\psi}{2}, \sin \frac{\psi}{2}\right),
\end{aligned}
$$

and set

$$
Q=y m(\psi), \quad P=x n(\psi) .
$$

Clearly, the point of $\Gamma$ determined by $\psi, x, y$ with $\psi \in[0,4 \pi), x, y \in \mathbb{R}$, lies in $\tilde{\Gamma}$; however, each point of $\tilde{\Gamma}$ is obtained twice in this way, since $(\psi, x, y)$ and $(\psi+2 \pi,-x,-y)$ determine the same point.

In terms of $r, \alpha, \beta$ we have

$$
\begin{aligned}
Q_{1} & =-\sqrt{2} r \sin \frac{\alpha+\beta}{2} \sin \frac{\alpha-\beta}{2}, \\
Q_{2} & =\sqrt{2} r \cos \frac{\alpha+\beta}{2} \sin \frac{\alpha-\beta}{2}, \\
P_{1} & =\sqrt{2} r \cos \frac{\alpha+\beta}{2} \cos \frac{\alpha-\beta}{2}, \\
P_{2} & =\sqrt{2} r \sin \frac{\alpha+\beta}{2} \cos \frac{\alpha-\beta}{2},
\end{aligned}
$$

hence

$$
\psi=\alpha+\beta, x=\sqrt{2} r \cos \frac{\alpha-\beta}{2}, y=\sqrt{2} r \sin \frac{\alpha-\beta}{2} .
$$

\section{$2.2 \quad$ Orbits}

The orbits are solutions to the system of differential equations

$$
\dot{Q}=\{Q, C\}=Q, \quad \dot{P}=\{P, C\}=P,
$$

which is easy to integrate:

$$
Q(t)=Q(0) e^{t}, \quad P(t)=P(0) e^{-t} .
$$

This implies immediately

$$
\psi(t)=\psi(0), x(t)=x(0) e^{-t}, y(t)=y(0) e^{t},
$$

hence $\psi$ and the product $x y$ is constant along orbits.

There are two disconnected sets of 'free' orbits (they leave definitely any compact set for sufficiently large or small $t$ ): 
I. $x y>0$, i.e. $x>0, y>0, \psi \in[0,4 \pi)$

(identical with $x<0, y<0, \psi \in[0,4 \pi)$ ),

and

II. $x y<0$, i.e. $x>0, y<0, \psi \in[0,4 \pi)$

(identical with $x<0, y>0, \psi \in[0,4 \pi)$ ).

They are separated by two-dimensional boundaries formed by 'imprisoned' orbits

III. $x=0, \psi \in[0,4 \pi)$,

and

IV. $y=0, \psi \in[0,4 \pi)$.

Observe that the space of orbits ' $\tilde{\Gamma}$ /orbits' is not Hausdorff and that the orbits with $x=0$ are not 'separable' from those with $y=0$. There will be, therefore, no global transversal surface [7].

\subsection{Perennials}

We have seen that the variables $Q_{i}, P_{i}$ satisfy the equations

$$
\left\{Q_{i}, C\right\}=Q_{i}, \quad\left\{P_{i}, C\right\}=-P_{i} .
$$

Thus we can guess the simplest functions which have vanishing Poisson brackets with $C$ :

$$
\begin{gathered}
Q_{1} P_{1},-Q_{2} P_{2}, Q_{1} P_{2}, Q_{2} P_{1}, \\
-\frac{Q_{1}}{Q_{2}}, \frac{P_{2}}{P_{1}} .
\end{gathered}
$$

The regular quadratic expressions (9) will be seen to be related to linear continuous symmetries, whereas the singular functions (10) are connected with the angular variable $\psi$.

Since the angular variable $\psi$ on $\tilde{\Gamma}$ is constant along $C$-orbits, it is another natural candidate for a symmetry generator. As an angular variable, it is however singular at its 'axis', so it is not a well-defined function on the whole of $\tilde{\Gamma}$. We can still find some extensions of $\psi$ to the outside of $\tilde{\Gamma}$. Such extensions are not unique; indeed, using the relations (5) - (8), we can immediately define the following two:

1. A function $\psi_{1}$, independent of $Q_{1}$ and $Q_{2}$, defined by

$$
\begin{aligned}
& \psi_{1}\left(P_{1}, P_{2}\right)=2 \arctan \frac{P_{2}}{P_{1}} \text { for } P_{1} \geq 0, \\
& \psi_{1}\left(P_{1}, P_{2}\right)=2 \pi+2 \arctan \frac{P_{2}}{P_{1}} \quad \text { for } P_{1} \leq 0 ;
\end{aligned}
$$


the 'axis ${ }_{1}$ ' of $\psi_{1}$ is the $Q$-plane $\left(P_{1}=P_{2}=0\right)$ and the corresponding cut $_{1}$ is given by $P_{1}=0, P_{2} \leq 0$.

2. A function $\psi_{2}$, independent of $P_{1}$ and $P_{2}$, defined by

$$
\begin{aligned}
& \psi_{2}\left(Q_{1}, Q_{2}\right)=-2 \arctan \frac{Q_{1}}{Q_{2}} \quad \text { for } Q_{2} \geq 0, \\
& \psi_{2}\left(Q_{1}, Q_{2}\right)=2 \pi-2 \arctan \frac{Q_{1}}{Q_{2}} \quad \text { for } Q_{2} \leq 0,
\end{aligned}
$$

the 'axis 2 ' of $\psi_{2}$ is the $P$-plane $\left(Q_{1}=Q_{2}=0\right)$ and $\psi_{2}$ is well-defined only on $\Gamma \backslash \mathrm{cut}_{2}$, where cut $_{2}$ is defined by $Q_{2}=0, Q_{1} \geq 0$; limits of $\psi_{2}$ towards the cut $_{2}$ from $\left(Q_{2}>0\right)$-side give $\psi_{2}=-\pi$, those from $\left(Q_{2}<0\right)$-side give $\psi_{2}=3 \pi$.

Further, for any smooth periodic function $f$ with the period $4 \pi, f\left(\psi_{\lambda}\right), \lambda=1,2$, is a smooth function on $\Gamma \backslash$ ' $\operatorname{axis}_{\lambda}$ '. The Hamiltonian vector field $\xi_{f}^{\lambda}$ of $f\left(\psi_{\lambda}\right)$ is given by $\xi_{f}^{\lambda}=f^{\prime}\left(\psi_{\lambda}\right) \xi^{\lambda}$, where $\xi^{\lambda}$ is the Hamiltonian vector field of $\psi_{\lambda} ; \xi^{\lambda}$ is well-defined on $\Gamma \backslash$ ' $\operatorname{axis}_{\lambda}$ ', as $d \psi_{\lambda}$ is ( $d \psi_{\lambda}$ is a closed but non-exact 1 -form).

We easily calculate $\xi^{\lambda}$ which results in

$$
\xi^{1}=-\frac{2 P_{2}}{P_{1}^{2}+P_{2}^{2}} \frac{\partial}{\partial Q_{1}}+\frac{2 P_{1}}{P_{1}^{2}+P_{2}^{2}} \frac{\partial}{\partial Q_{2}}
$$

and

$$
\xi^{2}=\frac{2 Q_{2}}{Q_{1}^{2}+Q_{2}^{2}} \frac{\partial}{\partial P_{1}}-\frac{2 Q_{1}}{Q_{1}^{2}+Q_{2}^{2}} \frac{\partial}{\partial P_{2}} .
$$

Thus, at $\tilde{\Gamma}, \xi^{1}$ and $\xi^{2}$ are tangent to $\tilde{\Gamma}, \xi^{\lambda}$ is singular at axis ${ }_{\lambda}$ for $\lambda=1,2$, but it is complete in $\Gamma \backslash^{\prime} \operatorname{axis}_{\lambda}$ '!

\section{$3 \quad$ Linear symmetries}

In this section, we will select a particular group of symmetries that is admitted by our system; it consists of linear transformations in the phase space $\Gamma$.

From the algebraic point of view, all linear canonical transformations of $\Gamma$ are given by the linear action of the symplectic group $S p(4, \mathbb{R})$ on $\mathbb{R}^{4}$. Of these, only those are symmetries which preserve the quadratic constraint $C=0$ with signature $(+--+)$.

In order to find all linear symmetries we first look at the intersection of $4 \times 4$ matrix groups $S p(4, \mathbb{R})$ and $O(2,2)$ acting on $\mathbb{R}^{4}$. In the coordinates $\mathbf{X}=\left(x_{n}\right)=$ $\left(Q_{1}, Q_{2}, P_{1}, P_{2}\right)$, the conditions on a $4 \times 4$ matrix $\boldsymbol{\Lambda}$ to be in $S p(4, \mathbb{R})$ as well as in $O(2,2)$ are

$$
\boldsymbol{\Lambda}^{T} \boldsymbol{\Omega} \boldsymbol{\Lambda}=\boldsymbol{\Omega} \quad \text { and } \quad \boldsymbol{\Lambda}^{T} \mathbf{G} \boldsymbol{\Lambda}=\mathbf{G}
$$


where $\boldsymbol{\Lambda}^{T}$ is the transposed matrix, and

$$
\boldsymbol{\Omega}=\left(\begin{array}{rr}
0 & I \\
-I & 0
\end{array}\right), \quad \mathbf{G}=\left(\begin{array}{cc}
0 & I \\
I & 0
\end{array}\right),
$$

with $I$ being the $2 \times 2$ unit matrix. Writing $\boldsymbol{\Lambda}$ in the block form, one can solve eqs. (11) with the result

$$
\Lambda=\left(\begin{array}{rr}
\Lambda & 0 \\
0 & \Lambda^{-1^{T}}
\end{array}\right)
$$

for all $\Lambda \in G L(2, \mathbb{R})$.

Since, because of (四), one should assume the preservation of $C$ only up to a factor $\gamma \neq 0$, eqs. (11) can be generalized into equations

$$
\boldsymbol{\Lambda}^{T} \boldsymbol{\Omega} \boldsymbol{\Lambda}=\boldsymbol{\Omega} \quad \text { and } \quad \boldsymbol{\Lambda}^{T} \mathbf{G} \boldsymbol{\Lambda}=\gamma \mathbf{G} .
$$

It turns out, however, that these have solutions only for $\gamma=1$ and $\gamma=-1$. In the latter case

$$
\boldsymbol{\Lambda}=\left(\begin{array}{rr}
0 & I \\
-I & 0
\end{array}\right)\left(\begin{array}{rr}
\Lambda & 0 \\
0 & \Lambda^{-1^{T}}
\end{array}\right), \quad \forall \Lambda \in G L(2, \mathbb{R}) .
$$

These results suggest that we investigate the continuous linear symmetries $\Lambda \in$ $G L^{+}(2, \mathbb{R})$ first, and then turn to an extension by discrete linear symmetries.

\subsection{Continuous linear symmetries}

Writing the group element in the form $\boldsymbol{\Lambda}=\exp \mathbf{A}$, the conditions for $\mathbf{A}$ to belong to the intersection of the corresponding Lie algebras $s p(4, \mathbb{R})$ and $o(2,2)$ become

$$
\mathbf{A}^{T} \boldsymbol{\Omega}+\boldsymbol{\Omega} \mathbf{A}=\mathbf{0}, \quad \mathbf{A}^{T} \mathbf{G}+\mathbf{G A}=\mathbf{0} .
$$

By straightforward calculation we obtain the general element $\mathbf{A}$ of $\operatorname{sp}(4, R) \cap o(2,2)$ of the form

$$
\mathbf{A}=\left(\begin{array}{rr}
A & 0 \\
0 & -A^{T}
\end{array}\right)
$$

where $A$ is an arbitrary real $2 \times 2$ matrix. Thus $\mathbf{A}$ can be expanded into the real linear combination

$$
\mathbf{A}=c_{0} \mathbf{L}_{0}+c_{1} \mathbf{L}_{1}+c_{2} \mathbf{L}_{2}+c \mathbf{Z}
$$

of 4 particular tensor products of $I$ and the Pauli matrices $\sigma^{a}, a=1,2,3$ :

$$
\begin{aligned}
\mathbf{L}_{0} & =-\frac{i}{2} \sigma^{2} \otimes I, \\
\mathbf{L}_{1} & =\frac{1}{2} \sigma^{1} \otimes \sigma^{3}, \\
\mathbf{L}_{2} & =\frac{1}{2} \sigma^{3} \otimes \sigma^{3}, \\
\mathbf{Z} & =I \otimes \sigma^{3} .
\end{aligned}
$$


These matrices satisfy the commutation relations

$$
\left[\mathbf{L}_{0}, \mathbf{L}_{1}\right]=-\mathbf{L}_{2},\left[\mathbf{L}_{0}, \mathbf{L}_{2}\right]=\mathbf{L}_{1},\left[\mathbf{L}_{1}, \mathbf{L}_{2}\right]=\mathbf{L}_{0},\left[\mathbf{Z}, \mathbf{L}_{\kappa}\right]=\mathbf{0}
$$

of $g l(2, \mathbb{R})$.

The generators of the corresponding linear canonical transformations are bilinear functions of the form

$$
f^{\mathbf{A}}=-\frac{1}{2} \sum_{m n} x_{m}(\mathbf{\Omega} \mathbf{A})_{m n} x_{n} .
$$

Since

$$
\left\{f^{\mathbf{A}}, f^{\mathbf{B}}\right\}=f^{[\mathbf{A}, \mathbf{B}]},
$$

we have a canonical realization of the Lie algebra $g l(2, \mathbb{R})$ based on two pairs of canonical variables.

Let us next find a connection of the functions (16) with the perennials listed in the previous section. We note that the four functions (9) are all $C^{\infty}$ over $\Gamma$ and the first two of them satisfy

$$
Q_{1} P_{1}=-Q_{2} P_{2}+C,
$$

hence they are not independent functions on $\tilde{\Gamma}$. But (16) with (13) - (15) give the three independent linear combinations $f^{\mathbf{L}_{\kappa}}, \kappa=0,1,2$, which we shall denote $L_{\kappa}$; as generators of extended point transformations, they can be written in the simple form

$$
\begin{aligned}
& L_{0}=f^{\mathbf{L}_{0}}=\frac{1}{2}\left(Q_{1} P_{2}-Q_{2} P_{1}\right)=Q^{T} \frac{1}{2} i \sigma^{2} P, \\
& L_{1}=f^{\mathbf{L}_{1}}=\frac{1}{2}\left(Q_{1} P_{2}+Q_{2} P_{1}\right)=Q^{T} \frac{1}{2} \sigma^{1} P, \\
& L_{2}=f^{\mathbf{L}_{2}}=\frac{1}{2}\left(Q_{1} P_{1}-Q_{2} P_{2}\right)=Q^{T} \frac{1}{2} \sigma^{3} P .
\end{aligned}
$$

The central element $\mathbf{Z}$ of the symmetry algebra corresponds to the constraint function

$$
C=f^{\mathbf{Z}}=Q_{1} P_{1}+Q_{2} P_{2}=Q^{T} I P .
$$

In accordance with (17), the Poisson bracket algebra of the obtained perennials is

$$
\left\{L_{0}, L_{1}\right\}=-L_{2},\left\{L_{0}, L_{2}\right\}=L_{1},\left\{L_{1}, L_{2}\right\}=L_{0},\left\{C, L_{\kappa}\right\}=0 .
$$

The formulae (18) - (20) suggest the use of a simple (anti-)isomorphic representation of $s l(2, \mathbb{R})$ by real $2 \times 2$ matrices $\frac{1}{2} \tau_{\kappa}, \kappa=0,1,2$, where

$$
\tau_{0}=i \sigma^{2}=\left(\begin{array}{rr}
0 & 1 \\
-1 & 0
\end{array}\right), \tau_{1}=\sigma^{1}=\left(\begin{array}{ll}
0 & 1 \\
1 & 0
\end{array}\right), \tau_{2}=\sigma^{3}=\left(\begin{array}{rr}
1 & 0 \\
0 & -1
\end{array}\right),
$$


and

$$
f^{\mathbf{L}_{\kappa}}=Q^{T} \frac{1}{2} \tau_{\kappa} P
$$

We shall not consider $f^{\mathbf{Z}}$ (represented by $I$ ) any more, since it generates the orbits. Thus, we restrict $g l(2, \mathbb{R})$ to $g l(2, \mathbb{R}) / \mathbb{R}=\operatorname{sl}(2, \mathbb{R})$.

At this point we arrived at the Lie algebra of the group $S L(2, \mathbb{R})$ and of its covering groups. In order to decide which of these groups is relevant as the symmetry of our model, we shall look at the one-parameter subgroups $\boldsymbol{\Lambda}_{\kappa}(t)$ generated by $\mathbf{L}_{\kappa}$, $\kappa=0,1,2$, and their action in $\Gamma$ and in the space of orbits in $\tilde{\Gamma}$. By exponentiation we find in the $2 \times 2$ representation

$$
\begin{aligned}
& \Lambda_{0}(t)=\exp \left(-\frac{i}{2} \sigma^{2} t\right)=I \cos \frac{t}{2}-i \sigma^{2} \sin \frac{t}{2} \\
& \Lambda_{1}(t)=\exp \left(\frac{\sigma^{1}}{2} t\right)=I \cosh \frac{t}{2}+\sigma^{1} \sinh \frac{t}{2} \\
& \Lambda_{2}(t)=\exp \left(\frac{\sigma^{3}}{2} t\right)=I \cosh \frac{t}{2}+\sigma^{3} \sinh \frac{t}{2}
\end{aligned}
$$

In the phase space $\Gamma$, the actions of the one-parameter subgroups on the column vectors $\mathbf{X}$ with components $\left(Q_{1}, Q_{2}, P_{1}, P_{2}\right)$ are given by $4 \times 4$ matrices $\boldsymbol{\Lambda}_{\kappa}(t)=$ $\exp \left(t \mathbf{L}_{\kappa}\right)$ :

$$
\begin{aligned}
& \boldsymbol{\Lambda}_{0}(t)=\mathbf{1} \cos \frac{t}{2}+\operatorname{diag}\left(-i \sigma^{2},-i \sigma^{2}\right) \sin \frac{t}{2} \\
& \boldsymbol{\Lambda}_{1}(t)=\mathbf{1} \cosh \frac{t}{2}+\operatorname{diag}\left(\sigma^{1},-\sigma^{1}\right) \sinh \frac{t}{2} \\
& \boldsymbol{\Lambda}_{2}(t)=\mathbf{1} \cosh \frac{t}{2}+\operatorname{diag}\left(\sigma^{3},-\sigma^{3}\right) \sinh \frac{t}{2}
\end{aligned}
$$

The integral curves of the actions generated by $\mathbf{L}_{\kappa}$ through a point $\mathbf{X}(0)$ in $\Gamma$ are then given by

$$
\mathbf{X}(t)=\boldsymbol{\Lambda}_{\kappa}(t) \mathbf{X}(0)
$$

Later also the following relation will be useful:

$$
\boldsymbol{\Lambda}_{\theta}(t)=\boldsymbol{\Lambda}_{0}(-\theta) \boldsymbol{\Lambda}_{1}(t) \boldsymbol{\Lambda}_{0}(\theta)=\exp \left[\left(\mathbf{L}_{1} \cos \theta+\mathbf{L}_{2} \sin \theta\right) t\right]
$$

Since

$$
\boldsymbol{\Lambda}_{0}(2 \pi)=-\mathbf{1}, \quad \boldsymbol{\Lambda}_{0}(4 \pi)=\boldsymbol{\Lambda}_{0}(0)=\mathbf{1},
$$

we see that the linear action of $S L(2, \mathbb{R})$ in $\Gamma$ is, in fact, a faithful representation of $S L(2, \mathbb{R})$ - the double covering $S_{2}(2,1)$ of $S O(2,1)$.

We have still to describe the action of the group $S L(2, \mathbb{R})$ on $\tilde{\Gamma}$ and on the orbits. We recall here that the perennials $L_{\kappa}$ are given by

$$
L_{0}=-\frac{1}{2} x y, \quad L_{1}=\frac{1}{2} x y \cos \psi, \quad L_{2}=-\frac{1}{2} x y \sin \psi .
$$


The real matrix ([12) corresponds to the $S L(2, \mathbb{R})$-transformation

$$
Q^{\prime}=\Lambda Q, \quad P^{\prime}=\Lambda^{-1^{T}} P
$$

where we put

$$
\Lambda=\left(\begin{array}{cc}
\alpha & \beta \\
\gamma & \delta
\end{array}\right), \quad \alpha \delta-\beta \gamma=1
$$

Substituting this into the definitions (2) - (4) of $x, y$ and $\psi$, one finds that

$$
y^{\prime} m\left(\psi^{\prime}\right)=y u, \quad x^{\prime} n\left(\psi^{\prime}\right)=x v,
$$

where

$$
\begin{aligned}
u & =\Lambda m(\psi)=\left(-\alpha \sin \frac{\psi}{2}+\beta \cos \frac{\psi}{2},-\gamma \sin \frac{\psi}{2}+\delta \cos \frac{\psi}{2}\right), \\
v & =\Lambda^{-1^{T}} n(\psi)=\left(-\gamma \sin \frac{\psi}{2}+\delta \cos \frac{\psi}{2}, \alpha \sin \frac{\psi}{2}-\beta \cos \frac{\psi}{2}\right) .
\end{aligned}
$$

The norms of the vectors $u$ and $v$ are equal. Denoting them by

$$
N(\psi, \Lambda)=\sqrt{\left(-\alpha \sin \frac{\psi}{2}+\beta \cos \frac{\psi}{2}\right)^{2}+\left(-\gamma \sin \frac{\psi}{2}+\delta \cos \frac{\psi}{2}\right)^{2}},
$$

we obtain

$$
y^{\prime}=y N(\psi, \Lambda), \quad x^{\prime}=x N(\psi, \Lambda)
$$

The angle $\psi^{\prime}$ is a function of $\psi$ and $\Lambda$ only, namely

$$
\psi^{\prime}=F(\psi, \Lambda)=2 \arctan \frac{\alpha \sin \frac{\psi}{2}-\beta \cos \frac{\psi}{2}}{-\gamma \sin \frac{\psi}{2}+\delta \cos \frac{\psi}{2}} .
$$

Thus, the transformations of $\psi$ decouple from those of $x$ and $y$. We observe that

$$
\frac{\partial F}{\partial \psi}(\psi, \Lambda)=\frac{1}{N^{2}(\psi, \Lambda)}
$$

The functions $F(\psi, \Lambda)$ and $N(\psi, \Lambda)$ satisfy certain composition laws. Namely, if

$$
y^{\prime \prime} m\left(\psi^{\prime \prime}\right)=y^{\prime} \Lambda^{\prime} m\left(\psi^{\prime}\right), \quad x^{\prime \prime} n\left(\psi^{\prime \prime}\right)=x^{\prime} \Lambda^{\prime} n\left(\psi^{\prime}\right)
$$

the substitution for $x^{\prime}, y^{\prime}$ and $\psi^{\prime}$ yields

$$
\begin{aligned}
y^{\prime \prime} & =y N(\psi, \Lambda) N\left(F(\psi, \Lambda), \Lambda^{\prime}\right), \\
x^{\prime \prime} & =x N(\psi, \Lambda) N\left(F(\psi, \Lambda), \Lambda^{\prime}\right), \\
\psi^{\prime \prime} & =F\left(F(\psi, \Lambda), \Lambda^{\prime}\right) .
\end{aligned}
$$


On the other hand, one has

$$
\begin{aligned}
y^{\prime \prime} & =y N\left(\psi, \Lambda^{\prime} \Lambda\right), \\
x^{\prime \prime} & =x N\left(\psi, \Lambda^{\prime} \Lambda\right), \\
\psi^{\prime \prime} & =F\left(\psi, \Lambda^{\prime} \Lambda\right) .
\end{aligned}
$$

Hence, we obtain that

$$
\begin{aligned}
N\left(\psi, \Lambda^{\prime} \Lambda\right) & =N(\psi, \Lambda) N\left(F(\psi, \Lambda), \Lambda^{\prime}\right) \\
F\left(\psi, \Lambda^{\prime} \Lambda\right) & =F\left(F(\psi, \Lambda), \Lambda^{\prime}\right) .
\end{aligned}
$$

In particular, for the subgroup

$$
\Lambda_{0}(t)=\left(\begin{array}{rr}
\cos \frac{t}{2} & -\sin \frac{t}{2} \\
\sin \frac{t}{2} & \cos \frac{t}{2}
\end{array}\right)
$$

we have

$$
F_{0}(\psi, t)=\psi+t, \quad N_{0}(\psi, t)=1 .
$$

For the subgroup

$$
\Lambda_{\theta}(t)=\left(\begin{array}{cc}
\cosh \frac{t}{2}+\sin \theta \sinh \frac{t}{2}, & \cos \theta \sinh \frac{t}{2} \\
\cos \theta \sinh \frac{t}{2}, & \cosh \frac{t}{2}-\sin \theta \sinh \frac{t}{2}
\end{array}\right),
$$

we find in this way

$$
F_{\theta}(\psi, t)=\frac{\pi}{2}-\theta+2 \arctan \left[e^{t} \tan \left(\frac{\psi+\theta}{2}-\frac{\pi}{4}\right)\right]
$$

for $\psi \in\left[-\frac{\pi}{2}-\theta, \frac{3 \pi}{2}-\theta\right]$,

$$
F_{\theta}(\psi, t)=\frac{5 \pi}{2}-\theta+2 \arctan \left[e^{t} \tan \left(\frac{\psi+\theta}{2}-\frac{\pi}{4}\right)\right]
$$

for $\psi \in\left[\frac{3 \pi}{2}-\theta, \frac{7 \pi}{2}-\theta\right]$, and

$$
N_{\theta}(\psi, t)=\sqrt{\cosh t-\sin (\psi+\theta) \sinh t} .
$$

For $\theta=0$ we get the function $F_{1}$ corresponding to $\Lambda_{1}(t)$,

$$
\begin{aligned}
& F_{1}(\psi, t)=\frac{\pi}{2}+2 \arctan \left[e^{t} \tan \left(\frac{\psi}{2}-\frac{\pi}{4}\right)\right] \text { for } \psi \in\left[-\frac{\pi}{2}, \frac{3 \pi}{2}\right], \\
& F_{1}(\psi, t)=\frac{5 \pi}{2}+2 \arctan \left[e^{t} \tan \left(\frac{\psi}{2}-\frac{\pi}{4}\right)\right] \text { for } \psi \in\left[\frac{3 \pi}{2}, \frac{7 \pi}{2}\right] .
\end{aligned}
$$


For $\theta=\frac{\pi}{2}$ we get 2 the function $F_{2}$ corresponding to $\Lambda_{2}(t)$,

$$
\begin{aligned}
& F_{2}(\psi, t)=2 \arctan \left(e^{t} \tan \frac{\psi}{2}\right) \text { for } \psi \in[-\pi, \pi], \\
& F_{2}(\psi, t)=2 \pi+2 \arctan \left(e^{t} \tan \frac{\psi}{2}\right) \text { for } \psi \in[\pi, 3 \pi] .
\end{aligned}
$$

We also have

$$
\frac{\partial F_{1}}{\partial t}=-\cos F_{1}, \quad \frac{\partial F_{2}}{\partial t}=\sin F_{2}
$$

The stratification of $\tilde{\Gamma}$ under the $S L(2, \mathbb{R})$-action is given by the following theorem.

Theorem. The $S L(2, \mathbb{R})$-orbits in $\tilde{\Gamma}$ are sets $\Gamma_{\alpha}$ given by

$$
x \cos \alpha+y \sin \alpha=0, \quad \alpha \in[0,2 \pi) .
$$

Proof. On the one hand, eqs. (23) imply

$$
x^{\prime} \cos \alpha+y^{\prime} \sin \alpha=0 \quad \text { iff } \quad x \cos \alpha+y \sin \alpha=0
$$

under all $\Lambda$. Thus the orbits of $S L(2, \mathbb{R})$ lie within $\Gamma_{\alpha}$. On the other hand, let $(x, y, \psi)$ and $\left(x^{\prime}, y^{\prime}, \psi^{\prime}\right)$ be two arbitrary points in $\Gamma_{\alpha}$. Then we can construct a transformation of $S L(2, \mathbb{R})$ transforming $(x, y, \psi)$ into $\left(x^{\prime}, y^{\prime}, \psi^{\prime}\right)$. First we observe that $x^{2}+y^{2}>0, x^{\prime 2}+y^{\prime 2}>0$, as the origin in $\tilde{\Gamma}$ has been excluded. Then the transformation is

$$
\Lambda_{0}\left(\psi^{\prime}-\pi\right) \Lambda_{2}\left(\log \frac{x^{\prime 2}+y^{\prime 2}}{x^{2}+y^{2}}\right) \Lambda_{0}(\pi-\psi) .
$$

Indeed, $\Lambda_{0}(\pi-\psi):(x, y, \psi) \mapsto(x, y, \pi)$. Next, applying $\Lambda_{2}(t)$ at $\psi=\pi$, the angle remains unchanged, while $x \mapsto x e^{\frac{t}{2}}$ and $y \mapsto y e^{\frac{t}{2}}$. The above choice of $t$ in $\Lambda_{2}$ yields $\left(x^{\prime}, y^{\prime}, \pi\right)$. Finally, we apply $\Lambda_{0}\left(\psi^{\prime}-\pi\right)$ to obtain $\left(x^{\prime}, y^{\prime}, \psi^{\prime}\right)$.

Suppose next that $\gamma_{1}$ and $\gamma_{2}$ are $C$-orbits. Does there exist an $S L(2, \mathbb{R})$-transformation $\Lambda$ such that

$$
\Lambda \gamma_{1}=\gamma_{2} ?
$$

The Theorem implies that $\boldsymbol{\Lambda}$ exists if and only if

a) $L_{0}>0$ along both $\gamma_{1}$ and $\gamma_{2}$;

$$
\begin{aligned}
&{ }^{2} \text { The functions } F_{\kappa}, \kappa=0,1,2 \text {, satisfy the relations } \\
& F_{i}(\psi, 0)=\psi, \\
& F_{i}\left(F_{i}(\psi, t), s\right)=F_{i}(\psi, t+s), \\
& F_{1}(\psi, t)=F_{2}\left(\psi-\frac{\pi}{2}, t\right)+\frac{\pi}{2} .
\end{aligned}
$$


b) $L_{0}<0$ along both $\gamma_{1}$ and $\gamma_{2}$;

c) $x=0$ along both $\gamma_{1}$ and $\gamma_{2}$;

d) $y=0$ along both $\gamma_{1}$ and $\gamma_{2}$.

Thus, $S L(2, \mathbb{R})$ does not act almost transitively on $\tilde{\Gamma}$ /orbits: there are four distinct $S L(2, \mathbb{R})$-orbits, and two of them [cases a) and b)] are open.

This property is quite general. Suppose that $\mathcal{G}$ is a connected group of regular symmetries (like $S L(2, \mathbb{R})$ ). Then, because of the regularity, no free orbit can be mapped into an imprisoned orbit. Suppose that the free orbits form several disconnected components. Then $\mathcal{G}$ cannot map $\gamma$ from one component to another one because of the connectivity.

It is interesting to compare the group theoretic and algebraic approaches at this point. The perennials $L_{0}, L_{1}$ and $L_{2}$ separate orbits in the open subset of $\tilde{\Gamma}$ defined by $L_{0} \neq 0$. Thus, from the algebraic point of view, they form a complete system. However, the group $S L(2, \mathbb{R})$ that is generated by them is not transitive!

Finally, the action of the group generated by $f\left(\psi_{\lambda}\right)$ on $\tilde{\Gamma}$ can be calculated using the form of $\xi^{\lambda}$ given at the end of subsection 2.3. We obtain easily

$$
\begin{aligned}
\xi^{1} x=0, & \xi^{2} x=\frac{2}{y}, \\
\xi^{1} y=\frac{2}{x}, & \xi^{2} y=0, \\
\xi^{1} \psi=0, & \xi^{2} \psi=0 .
\end{aligned}
$$

Thus $\xi^{\lambda}$ is complete on $\tilde{\Gamma} \backslash \operatorname{axis}_{\lambda}, \lambda=1,2$, axis $\lambda \cap \tilde{\Gamma}$ being given by $x=0$ for $\psi_{1}$ and by $y=0$ for $\psi_{2}$

\subsection{Discrete linear symmetries}

We have seen that the whole group of linear symmetries preserving $\mathbf{G}$ up to a nonvanishing multiplier, consists of two disconnected sets

$$
\left\{\left(\begin{array}{rr}
\Lambda & 0 \\
0 & \Lambda^{-1^{T}}
\end{array}\right)\right\} \quad \text { and }\left\{\left(\begin{array}{rr}
0 & I \\
-I & 0
\end{array}\right)\left(\begin{array}{rr}
\Lambda & 0 \\
0 & \Lambda^{-1^{T}}
\end{array}\right)\right\}
$$

with $\Lambda \in G L(2, \mathbb{R})$ which itself consists of two connected components, sgn $\operatorname{det} \Lambda=$ \pm 1 . Recall that the group $S L(2, \mathbb{R}$ ) of the nontrivial linear symmetries (which is a subgroup of the first component containing the identity) is not almost transitive. Let us therefore try to add some discrete transformations to it.

- One choice is the discrete transformation from (33)

$$
\tau=\left(\begin{array}{rr}
0 & I \\
-I & 0
\end{array}\right)(=\Omega)
$$


which reverses the sign of $C$ and allows us to extend $S L(2, \mathbb{R})$ by a disconnected component as in (33).

- Another choice is a transformation from the second component of $G L(2, R)$ (with negative determinant):

$$
\sigma=\operatorname{diag}\left(-\sigma^{3},-\sigma^{3}\right), \quad \operatorname{det} \sigma^{3}=-1
$$

The transformations act on the coordinates as follows:

$$
\begin{gathered}
\tau: Q_{1} \mapsto+P_{1}, Q_{2} \mapsto+P_{2}, P_{1} \mapsto-Q_{1}, P_{2} \mapsto-Q_{2}, \\
\sigma: Q_{1} \mapsto-Q_{1}, Q_{2} \mapsto+Q_{2}, P_{1} \mapsto-P_{1}, P_{2} \mapsto+P_{2},
\end{gathered}
$$

and satisfy the relations

$$
\sigma^{2}=\mathbf{1}, \quad \tau^{2}=-\mathbf{1}, \quad \tau \sigma=\sigma \tau
$$

Evidently, $\tau$ and $\sigma$ generate the Abelian group $Z_{4} \times Z_{2}$ of order 8. Also the actions of $\tau$ and $\sigma$ on $\tilde{\Gamma}$ can be found easily:

$$
\begin{aligned}
\tau: & x \mapsto y, \quad y \mapsto x, \quad \psi \mapsto \psi-\pi, \\
& (x \mapsto-y, \quad y \mapsto-x, \quad \psi \mapsto \psi+\pi), \\
\sigma: x \mapsto-x, \quad y \mapsto y, \quad \psi \mapsto-\psi, & \mapsto \mapsto-y \mapsto-y, \quad \psi \mapsto 2 \pi-\psi) .
\end{aligned}
$$

A straightforward calculation reveals the automorphisms of $\operatorname{sl}(2, \mathbb{R})$ induced by $\tau$ and $\sigma$ :

$$
\begin{aligned}
& \tau \mathbf{L}_{0} \tau^{-1}=+\mathbf{L}_{0}, \quad \tau \mathbf{L}_{1} \tau^{-1}=-\mathbf{L}_{1}, \quad \tau \mathbf{L}_{2} \tau^{-1}=-\mathbf{L}_{2}, \\
& \sigma \mathbf{L}_{0} \sigma^{-1}=-\mathbf{L}_{0}, \quad \sigma \mathbf{L}_{1} \sigma^{-1}=-\mathbf{L}_{1}, \quad \sigma \mathbf{L}_{2} \sigma^{-1}=+\mathbf{L}_{2} \text {. }
\end{aligned}
$$

In the adjoint representation of $S L(2, \mathbb{R}) \approx S O_{2}(2,1), \sigma$ corresponds to the inversion of the axis 2. The extended group generated by $\mathbf{L}_{0}, \mathbf{L}_{1}, \mathbf{L}_{2}$ and $\sigma$ can, therefore, be also regarded as $O_{2}^{+}(2,1)$, the orthochronous Lorentz group in three dimensions. The group $O_{2}^{+}(2,1)$ acts almost transitively on $\tilde{\Gamma}$ /orbit. Let us select this group as our first-class canonical group.

\section{Transversal surfaces}

We have seen in Sec. 2 that there is no global transversal surface. In the present paper, we will try to use instead a system of maximal transversal surfaces such that 
each orbit is intersected by at least one surface of the system. In this section, we describe an example of such a system.

Let us choose the transversal surfaces $\Gamma_{1}$ and $\Gamma_{2}$ by

$$
\begin{gathered}
\Gamma_{1}=\{(x, y, \psi) \in \tilde{\Gamma} \mid x=T, \quad-\infty<y<\infty, \quad 0 \leq \psi<4 \pi\}, \\
\Gamma_{2}=\{(x, y, \psi) \in \tilde{\Gamma} \mid y=T, \quad-\infty<x<\infty, \quad 0 \leq \psi<4 \pi\},
\end{gathered}
$$

$T$ being an arbitrary positive constant. The domain of $\Gamma_{1}$ is the subset $D\left(\Gamma_{1}\right)$ of $\tilde{\Gamma}$ satisfying $x \neq 0$, while the domain of $\Gamma_{2}$ is that satisfying $y \neq 0$. Thus, $\Gamma_{1}$ and $\Gamma_{2}$ together intersect all orbits. It is also clear that $\Gamma_{1}$ and $\Gamma_{2}$ are both maximal.

The topology of $\Gamma_{1}$ or $\Gamma_{2}$ is $\mathbb{R} \times S^{1}$. We can introduce coordinates $\left(l_{i}, \varphi_{i}\right)$ on $\Gamma_{i}$ by the following embedding formulae. For $\Gamma_{1}$ in $\tilde{\Gamma}$,

$$
x=T, \quad y=-\frac{2 l_{1}}{T}, \quad \psi=\varphi_{1}
$$

and in $\Gamma$,

$$
\begin{aligned}
Q_{1} & =\frac{2 l_{1}}{T} \sin \frac{\varphi_{1}}{2}, \quad Q_{2}=-\frac{2 l_{1}}{T} \cos \frac{\varphi_{1}}{2} \\
P_{1} & =T \cos \frac{\varphi_{1}}{2}, \quad P_{2}=T \sin \frac{\varphi_{1}}{2}
\end{aligned}
$$

for $\Gamma_{2}$ in $\tilde{\Gamma}$,

$$
x=-\frac{2 l_{2}}{T}, \quad y=T, \quad \psi=\varphi_{2}
$$

and in $\Gamma$,

$$
\begin{aligned}
& Q_{1}=-T \sin \frac{\varphi_{2}}{2}, \quad Q_{2}=T \cos \frac{\varphi_{2}}{2}, \\
& P_{1}=-\frac{2 l_{2}}{T} \cos \frac{\varphi_{2}}{2}, \quad P_{2}=-\frac{2 l_{2}}{T} \sin \frac{\varphi_{2}}{2},
\end{aligned}
$$

Then the corresponding pull-backs of the symplectic form $\Omega=d P_{1} \wedge d Q_{1}+d P_{2} \wedge d Q_{2}$ in $\Gamma$ are

$$
\Omega_{i}=d l_{i} \wedge d \varphi_{i}, \quad i=1,2 .
$$

Thus, $\left(\Gamma_{i}, \Omega_{i}\right)$ can be considered as cotangent bundles, $T^{*} \mathcal{C}_{i}$, with the Liouville forms $\theta_{i}=l_{i} d \varphi_{i}$, so that $\Omega_{i}=d \theta_{i}$ and $\mathcal{C}_{i} \approx S^{1}$.

Observe that the orbits in $D\left(\Gamma_{i}\right)$ can be distinguished by the values of two perennials, $L_{0}=-x y / 2$ and $\psi$. Their values on $\Gamma_{i}$ are given by

$$
\left.L_{0}\right|_{\Gamma_{i}}=l_{i},\left.\quad \psi\right|_{\Gamma_{i}}=\varphi_{i}
$$

Let us define the subsets $\Gamma_{i}^{ \pm}, \Gamma_{i}^{0}$ of $\Gamma_{i}$ as follows

$$
\begin{aligned}
\Gamma_{i}^{+} & =\left\{p \in \Gamma_{i} \mid l_{i}(p)>0\right\} \\
\Gamma_{i}^{0} & =\left\{p \in \Gamma_{i} \mid l_{i}(p)=0\right\} \\
\Gamma_{i}^{-} & =\left\{p \in \Gamma_{i} \mid l_{i}(p)<0\right\} .
\end{aligned}
$$


Each point $\left(l_{1}, \varphi_{1}\right)$ of $\Gamma_{1}^{+}$determines a unique orbit $\gamma$ with the values of $L_{0}=l_{1}$ and $\psi=\varphi_{1}$ along it. The orbit $\gamma$ intersects the surface $y=-T$ at the point

$$
x=\frac{2 l_{1}}{T}, \quad y=-T, \quad \psi=\varphi_{1},
$$

because $L_{0}=-\frac{1}{2} x y, x$ is negative at the intersection $\Gamma_{1} \cap \gamma$, and neither $x$ nor $y$ can change sign along an orbit. Thus, the intersection $\gamma \cap \Gamma_{2}$ has the coordinates

$$
x=-\frac{2 l_{1}}{T}, \quad y=T, \quad \psi=\varphi_{1}+2 \pi,
$$

which corresponds to

$$
l_{2}=l_{1}, \quad \varphi_{2}=\varphi_{1}+2 \pi .
$$

The mapping $\rho_{+}: \Gamma_{1}^{+} \rightarrow \Gamma_{2}^{+}$defined by eq. (36) is a symplectic diffeomorphism. Similarly, we obtain $\rho_{-}: \Gamma_{1}^{-} \rightarrow \Gamma_{2}^{-}$which is given by

$$
l_{2}=l_{1}, \quad \varphi_{2}=\varphi_{1} .
$$

We also introduce the combined map $\rho$ of $\Gamma_{1}^{+} \cup \Gamma_{1}^{-}$onto $\Gamma_{2}^{+} \cup \Gamma_{2}^{-}$; it will be called a pasting map.

We observe finally that none of the symplectic diffeomorphisms $\rho_{ \pm}$and $\rho$ can be considered as a "lift" 3 of a diffeomorphism between the configuration spaces $\mathcal{C}_{1}$ and $\mathcal{C}_{2}\left(\approx S^{1}\right)$. Thus, our pasting is rather different from that encountered in gauge theories with non-trivial fibre bundles, where patches of a configuration space are pasted together by gauge transformations [8, 9]. This is due to the fact that the transformation generated by constraints of gauge fields which are linear in momenta, leaves configuration space invariant, whereas our constraint is quadratic in momenta.

Next we will project the symmetry transformations to the transversal surfaces, as it is described in I.

Let $a_{i}(\Lambda), i=1,2$, denote the (non-linear) action of a symmetry $\Lambda: \Gamma \rightarrow \Gamma$ on $\Gamma_{i}$ (for the definitions see I; $a_{i}(\Lambda)$ are canonical transformations on $\left(\Gamma_{i}, \Omega_{i}\right)$ ). For the group $S L(2, \mathbb{R})$, we easily read off from the relations of Sec. 3.1 that

$$
a_{i}(\Lambda)\left(l_{i}, \varphi_{i}\right)=\left(l_{i} N^{2}\left(\varphi_{i}, \Lambda\right), F\left(\varphi_{i}, \Lambda\right)\right)
$$

Thus the sign of $l_{i}$ is not changed by any transformation of $S L(2, \mathbb{R})$ and the sets $\Gamma_{i}^{ \pm}$ and $\Gamma_{i}^{0}$ are $a_{i}(S L(2, \mathbb{R}))$-invariant. Moreover, these sets are orbits of $a_{i}(S L(2, \mathbb{R}))$; this follows easily from the Theorem of Sec. 3.1. In particular, the action $a_{i}$ of $S L(2, \mathbb{R})$ is transitive in each of the (overlapping) sets $\Gamma_{i}^{ \pm}$.

\footnotetext{
${ }^{3}$ Equivalently, $\rho_{ \pm}$and $\rho$ are canonical transformations which are not extended point transformations.
} 
Another important observation is that both sets $\Gamma_{1}^{+}$and $\Gamma_{2}^{+}$which are 'pasted together' by $\rho_{+}$have the property that $\left.L_{0}\right|_{\Gamma_{i}^{+}}=l_{i}>0, i=1,2$. Similarly, $\left.L_{0}\right|_{\Gamma_{i}^{-}}=l_{i}<0$. Now, $L_{0}$ as a function on $\Gamma_{i}$ generates, via Poisson brackets, the one-parameter group $a_{i}\left(\Lambda_{0}(t)\right)$ [1]. Thus, we will try to identify the relevant representations $R$ of $S L(2, \mathbb{R})$ by the sign of eigenvalues of the corresponding generator of $R\left(\Lambda_{0}(t)\right)$.

The action of the discrete transformation $\sigma$ on $\tilde{\Gamma}$ is given by (34). Thus, on $\Gamma_{1}$,

$$
a_{1}(\sigma): l_{1} \mapsto-l_{1}, \varphi_{1} \mapsto 2 \pi-\varphi_{1}
$$

and on $\Gamma_{2}$

$$
a_{2}(\sigma): l_{2} \mapsto-l_{2}, \varphi_{2} \mapsto-\varphi_{2}
$$

Note that both $a_{i}(\sigma)$ are extended point transformations. Moreover, the actions $a_{i}$ of $\mathrm{O}_{2}^{+}(2,1)$ (2 connected components) are almost transitive on $\Gamma_{i}$, the corresponding orbits being $\Gamma_{i}^{+} \cup \Gamma_{i}^{-}(2$ connected components $)$ and $\Gamma_{i}^{0}$.

The infinitesimal generators of the transformations (37) are easily calculated using the relations (22), (24) and (32):

$$
\begin{aligned}
d a_{i}\left(\frac{\tau_{0}}{2}\right) & =\frac{\partial}{\partial \varphi_{i}}, \\
d a_{i}\left(\frac{\tau_{1}}{2}\right) & =-\sin \varphi_{i} l_{i} \frac{\partial}{\partial l_{i}}-\cos \varphi_{i} \frac{\partial}{\partial \varphi_{i}}, \\
d a_{i}\left(\frac{\tau_{2}}{2}\right) & =-\cos \varphi_{i} l_{i} \frac{\partial}{\partial l_{i}}+\sin \varphi_{i} \frac{\partial}{\partial \varphi_{i}} ;
\end{aligned}
$$

they are global Hamiltonian vector fields of the following functions on $\Gamma_{i}$ :

$$
\begin{aligned}
L_{0}^{i} & =l_{i}, \\
L_{1}^{i} & =-l_{i} \cos \varphi_{i}, \\
L_{2}^{i} & =l_{i} \sin \varphi_{i} .
\end{aligned}
$$

The functions $L_{\kappa}^{i}$ are just the restrictions of the original generators $L_{\kappa}$ to $\Gamma_{i}$ (cf. (18) $-(20))$.

To summarize: we have reduced the original constrained system to two overlapping unconstrained systems $\mathcal{S}_{1}$ and $\mathcal{S}_{2}$ living on the reduced phase spaces $\Gamma_{1}$ and $\Gamma_{2}$,

$$
\Gamma_{i} \approx T^{*} S^{1}=\left\{\left(l_{i}, \varphi_{i}\right) \mid l_{i} \in \mathbb{R}, \varphi_{i} \in[0,4 \pi)\right\},
$$

with the symplectic forms $\Omega_{i}=d l_{i} \wedge d \varphi_{i}$. The perennials of the original system define observables for $S_{1}$ and $S_{2}$ by projection to the surfaces $\Gamma_{1}$ and $\Gamma_{2}$ as described in I:

$$
L_{0}^{i}=l_{i}, \quad L_{1}^{i}=-l_{i} \cos \varphi_{i}, \quad L_{2}^{i}=l_{i} \sin \varphi_{i}
$$


and the parity

$$
a_{1}(\sigma): l_{1} \mapsto-l_{1}, \varphi_{1} \mapsto 2 \pi-\varphi_{1}, \quad a_{2}(\sigma): l_{2} \mapsto-l_{2}, \varphi_{2} \mapsto-\varphi_{2}
$$

The perennial $f\left(\psi_{\lambda}\right)(\lambda=1,2)$, where $f$ is any $4 \pi$-periodic function of its argument, can be projected only to the transaversal surface $\Gamma_{\lambda}(i=\lambda)$. The projection is given by

$$
\left.f\left(\psi_{\lambda}\right)\right|_{\Gamma_{\lambda}}=f\left(\psi_{\lambda}\right), \quad(i=\lambda) \quad \lambda=1,2 .
$$

We stress that there is no differentiable projection of $f\left(\psi_{1}\right)$ to $\Gamma_{2}$ or of $f\left(\psi_{2}\right)$ to $\Gamma_{1}$ !

There is a relation among the classical observables, $L_{0}^{i}, L_{1}^{i}$ and $L_{2}^{i}$ :

$$
-\left(L_{0}^{i}\right)^{2}+\left(L_{1}^{i}\right)^{2}+\left(L_{2}^{i}\right)^{2}=0 .
$$

These and $a_{i}(\sigma)$ generate the group $O_{2}^{+}(2,1)$.

The overlapping between $S_{1}$ and $S_{2}$ is defined by two maps:

$$
\rho_{+}: \Gamma_{1}^{+} \rightarrow \Gamma_{2}^{+}, \quad l_{1}>0, l_{2}>0
$$

given by the functions

$$
l_{2}\left(l_{1}, \varphi_{1}\right)=l_{1}, \quad \varphi_{2}\left(l_{1}, \varphi_{1}\right)=\varphi_{1}+2 \pi
$$

and

$$
\rho_{-}: \Gamma_{1}^{-} \rightarrow \Gamma_{2}^{-}, \quad l_{1}<0, l_{2}<0
$$

given by the functions

$$
l_{2}\left(l_{1}, \varphi_{1}\right)=l_{1}, \quad \varphi_{2}\left(l_{1}, \varphi_{1}\right)=\varphi_{1}
$$

\section{Canonical quantization}

The next step in the group quantization method is to find, for systems $\mathcal{S}_{i}$, suitable unitary representations of the group $\mathrm{O}_{2}^{+}(2,1)$ defining the corresponding Hilbert spaces $\mathcal{H}_{i}$ and quantum observables as hermitian operators in $\mathcal{H}_{i}$.

Here we study the representations of the group that can be found by a straightforward application of canonical quantization method to the reduced systems $\mathcal{S}_{i}$. Thus, as our Hilbert spaces we choose the spaces of square-integrable functions on the configuration space $S^{1}$,

$$
\overline{\mathcal{H}_{i}}=L^{2}\left(S^{1}, d \varphi_{i}\right),
$$

${ }^{4}$ The theory of representations of the group $S L(2, \mathbb{R})$ and its covering groups can be found, e.g. in [10, 11] and [12]. 
i.e. with the inner product

$$
(\Psi, \Phi)_{i}=\frac{1}{4 \pi} \int_{0}^{4 \pi} \bar{\Psi}\left(\varphi_{i}\right) \Phi\left(\varphi_{i}\right) d \varphi_{i} .
$$

An obvious symmetrization gives the hermitian operators for the observables $L_{\kappa}^{i}$,

$$
\begin{aligned}
\hat{L}_{0}^{i} & =-i \frac{\partial}{\partial \varphi_{i}}, \\
\hat{L}_{1}^{i} & =i \cos \varphi_{i} \frac{\partial}{\partial \varphi_{i}}-\frac{i}{2} \sin \varphi_{i}, \\
\hat{L}_{2}^{i} & =-i \sin \varphi_{i} \frac{\partial}{\partial \varphi_{i}}-\frac{i}{2} \cos \varphi_{i} .
\end{aligned}
$$

We show that the corresponding unitary representation of $S L(2, \mathbb{R})$ is given by

$$
[U(\Lambda) \Psi](\varphi)=\frac{1}{N(\varphi, \Lambda)} \Psi(F(\varphi, \Lambda)) .
$$

$U(\Lambda)$ is unitary, since

$$
\begin{aligned}
(U(\Lambda) \Psi, U(\Lambda) \Phi) & =\frac{1}{4 \pi} \int_{0}^{4 \pi} \frac{1}{N^{2}(\varphi, \Lambda)} \bar{\Psi}(F(\varphi, \Lambda)) \Phi(F(\varphi, \Lambda)) d \varphi \\
& =\frac{1}{4 \pi} \int_{F(0, \Lambda)}^{F(4 \pi, \Lambda)} \bar{\Psi}(F) \Phi(F) d F=(\Psi, \Phi)
\end{aligned}
$$

due to (25). For the composition of two group elements $\Lambda, \Lambda^{\prime}$ we obtain

$$
\begin{aligned}
{\left[U\left(\Lambda^{\prime}\right)(U(\Lambda) \Psi)\right](\varphi) } & =U\left(\Lambda^{\prime}\right) \frac{1}{N(\varphi, \Lambda)} \Psi(F(\varphi, \Lambda)) \\
& =\frac{1}{N\left(\varphi, \Lambda^{\prime}\right)} \frac{1}{N\left(F\left(\varphi, \Lambda^{\prime}\right), \Lambda\right)} \Psi\left(F\left(F\left(\varphi, \Lambda^{\prime}\right), \Lambda\right)\right) \\
& =\frac{1}{N\left(\varphi, \Lambda \Lambda^{\prime}\right)} \Psi\left(F\left(\varphi, \Lambda \Lambda^{\prime}\right)\right)=\left(U\left(\Lambda \Lambda^{\prime}\right) \Psi\right)(\varphi)
\end{aligned}
$$

because of (26) and (27). Finally, specializing to the subgroups $\Lambda_{0}(t)$ and $\Lambda_{\theta}(t)$ we find that

$$
\begin{aligned}
\left(U\left(\Lambda_{0}(t)\right) \Psi\right)(\varphi) & =\Psi(\varphi+t), \\
\left(U\left(\Lambda_{\theta}(t)\right) \Psi\right)(\varphi) & =\frac{1}{N_{\theta}(\varphi+t)} \Psi\left(F_{\theta}(\varphi+t)\right) .
\end{aligned}
$$

The $t$-derivatives of these functions at $t=0$ are

$$
\begin{aligned}
i \hat{L}_{0} \Psi(\varphi) & =\left.\frac{d}{d t}\left[U\left(\Lambda_{0}(t)\right) \Psi\right](\varphi)\right|_{t=0}=\Psi^{\prime}(\varphi), \\
i \hat{L}_{\theta} \Psi(\varphi) & =\left.\frac{d}{d t}\left[U\left(\Lambda_{0}(t)\right) \Psi\right](\varphi)\right|_{t=0} \\
& =-\cos (\varphi+\theta) \Psi^{\prime}(\varphi)+\frac{1}{2} \sin (\varphi+\theta) \Psi(\varphi),
\end{aligned}
$$


which finishes the proof, since the result is in accordance with (41) - (43). Observe that $S L(2, \mathbb{R})$ acts from the right!

As $a_{i}(\sigma)$ are extended point transformations whose projections on the configuration spaces $\mathcal{C}_{i}$ leave the integration measure invariant, we can choose for the corresponding operators $\hat{\sigma}_{i}$

$$
\begin{aligned}
\left(\hat{\sigma}_{1} \Psi\right)\left(\varphi_{1}\right) & =\Psi\left(2 \pi-\varphi_{1}\right), & \varphi_{1} \in[0,2 \pi), \\
& =\Psi\left(6 \pi-\varphi_{1}\right), & \varphi_{1} \in[2 \pi, 4 \pi), \\
\left(\hat{\sigma}_{2} \Psi\right)\left(\varphi_{2}\right) & =\Psi\left(4 \pi-\varphi_{2}\right), & \varphi_{2} \in[0,4 \pi) .
\end{aligned}
$$

It is easy to verify that the commutation relations correponding to eqs. (21) and (35) are satisfied. Thus, we have hermitian representations of the Lie algebra and with it unitary representations of the group $O_{2}^{+}(2,1)$ in $\overline{\mathcal{H}}_{i}$.

\subsection{Representations of $\mathrm{O}_{2}^{+}(2,1)$}

We want to clarify which of the known representations of $O_{2}^{+}(2,1)$ we have (cf. [10, [11 and [12), as well as if the representations are faithful and irreducible. Taking the eigenfunctions of $\hat{L}_{0}^{i}$

$$
\hat{L}_{0}^{i} \Psi_{m}=\frac{m}{2} \Psi_{m}, \quad \Psi_{m}\left(\varphi_{i}\right)=e^{\frac{i}{2} m \varphi_{i}}, \quad m \in Z,
$$

where $Z$ is the set of all integers, as suitable basis in $\overline{\mathcal{H}}_{i}$, we find for the shift operators

$$
\begin{array}{r}
\hat{L}_{ \pm}^{i}=\hat{L}_{1}^{i} \mp i \hat{L}_{2}^{i}=i e^{ \pm \frac{i \varphi_{i}}{2}} \frac{\partial}{\partial \varphi_{i}} e^{ \pm \frac{i \varphi_{i}}{2}} \\
\hat{L}_{ \pm}^{i} \Psi_{m}=-\frac{1}{2}(m \pm 1) \Psi_{m \pm 2} .
\end{array}
$$

Suppose that $m$ is even. Then

$$
\hat{L}_{ \pm}^{i} \Psi_{m} \neq 0
$$

and all such functions span one irreducible representation. The representation space $\mathcal{H}_{i}^{0} \subset \overline{\mathcal{H}}_{i}$ contains all $2 \pi$-periodic elements of $\overline{\mathcal{H}}_{i}$. Comparison with 12 reveals that the representation of $S L(2, \mathbb{R})$ realized in $\mathcal{H}_{i}^{0}$ is $C_{-\frac{1}{4}}^{0}$ belonging to the principal series (with the value of the Casimir operator $q=-\frac{1}{4}$ ).

Suppose now that $m$ is odd. Then

$$
\begin{array}{ll}
\hat{L}_{+}^{i} \Psi_{m}=0 & \text { for } m=-1, \\
\hat{L}_{-}^{i} \Psi_{m}=0 & \text { for } m=+1 .
\end{array}
$$

Hence we obtain two irreducible representations of $S L(2, \mathbb{R})$, one spanned by the functions $\Psi_{m}$ for $m=-1,-2, \ldots$, and the other for $m=1,2, \ldots$. Let us denote 
the corresponding subspaces of $\overline{\mathcal{H}}_{i}$ by $\mathcal{H}_{i}^{+}$and $\mathcal{H}_{i}^{-}$, respectively. The corresponding representations $R_{i}^{+}=D_{\frac{1}{2}}^{-}$and $R_{i}^{-}=D_{\frac{1}{2}}^{+}$belong to the discrete series (the value of the Casimir operator is again $q=-\frac{1}{4}$ [12].)

As a result we obtained the orthogonal direct sum decomposition

$$
\overline{\mathcal{H}}_{i}=\mathcal{H}_{i}^{0} \oplus \mathcal{H}_{i}^{+} \oplus \mathcal{H}_{i}^{-}
$$

and observe that, of the three irreducible representations of $S L(2, \mathbb{R})$, only $C_{-\frac{1}{4}}^{0}$ in $\mathcal{H}_{i}^{0}$ is not faithful, as the rotation by $2 \pi, \varphi_{i} \mapsto \varphi_{i}+2 \pi$, is represented by the identity. Thus we have to throw the even $m$ subspace $\mathcal{H}_{i}^{0}$ away.

Consider now the action of the parity operators $\hat{\sigma}_{i}$ on the basis functions $\Psi_{m}$ with $m$ odd. We obtain

$$
\hat{\sigma}_{1} \Psi_{m}=-\Psi_{-m}, \quad \hat{\sigma}_{2} \Psi_{m}=\Psi_{-m} .
$$

Thus the operators $\hat{\sigma}_{i}$ map $\mathcal{H}_{i}^{+}$onto $\mathcal{H}_{i}^{-}$and vice versa, so the group $O_{2}^{+}(2,1)$ is represented irreducibly and faithfully on

$$
\mathcal{H}_{i}:=\mathcal{H}_{i}^{+} \oplus \mathcal{H}_{i}^{-} .
$$

The elements of $\mathcal{H}_{i}$ are all $2 \pi$-antiperiodic functions of $L^{2}\left(S^{1}\right)$.

We have found the two irreducible representations by canonical quantization; let us close this section by looking to see whether or not they satisfy the principles of algebraic and group quantization.

1. Are all subalgebras of elementary observables represented irreducibly? This is not the case, as $\hat{L}_{\kappa}^{i}, \kappa=0,1,2$, form such a subalgebra, and $\mathcal{H}_{i}$ is reducible. Observe that any of the three Hilbert spaces $\mathcal{H}_{i}^{0}, \mathcal{H}_{i}^{+}$and $\mathcal{H}_{i}^{-}$is a possible choice for the algebraic quantization.

2. Are all relations satisfied?

Not quite! If we apply the prescription given in I (cf. also [3]) we obtain that $-\left(\hat{L}_{0}^{i}\right)^{2}+\left(\hat{L}_{1}^{i}\right)^{2}+\left(\hat{L}_{2}^{i}\right)^{2}=\frac{1}{4}$.

3. Are all canonical subgroups represented irreducibly?

They are! Indeed, we have the (minimal) canonical group $O_{2}^{+}(2,1)$ and in the spaces $\mathcal{H}_{i}$ an irreducible representation is realized.

\section{$6 \quad$ Pasting the Hilbert spaces}

In the previous section, we have obtained two quantum theories: one for each of the reduced systems $S_{1}$ and $S_{2}$ that correspond to the two maximal transversal surfaces 
$\Gamma_{1}$ and $\Gamma_{2}$. In the present section, we try to construct a single quantum theory from the two.

Let us compare the action of the group $S L(2, \mathbb{R})$ in the classical phase spaces $\Gamma_{i}$, with the representations of $S L(2, \mathbb{R})$ in the quantum Hilbert spaces $\mathcal{H}_{1}$ and $\mathcal{H}_{2}$. First, the phase spaces have submanifolds $\Gamma_{i}^{+}$and $\Gamma_{i}^{-}$such that $S L(2, \mathbb{R})$ acts transitively inside each of them. Similarly, $\mathcal{H}_{i}$ has two subspaces $\mathcal{H}_{i}^{ \pm}$in which the representations $R_{i}^{ \pm}$of $S L(2, \mathbb{R})$ are irreducible. Second, there are pasting maps, $\rho_{+}: \Gamma_{1}^{+} \rightarrow \Gamma_{2}^{+}$and $\rho_{-}: \Gamma_{1}^{-} \rightarrow \Gamma_{2}^{-}$which are symplectic and equivariant with the action of $S L(2, \mathbb{R})$. That is, let $a_{i}^{ \pm}(\Lambda): \Gamma_{i}^{ \pm} \rightarrow \Gamma_{i}^{ \pm}, \Lambda \in S L(2, \mathbb{R})$, be the corresponding maps in $\Gamma_{i}^{ \pm}$; then

$$
a_{2}^{ \pm}(\Lambda)=\rho_{ \pm} \circ a_{1}^{ \pm}(\Lambda) \circ \rho_{ \pm}^{-1} .
$$

We can ask for quantum pasting maps, $U_{ \pm}: \mathcal{H}_{1}^{ \pm} \rightarrow \mathcal{H}_{2}^{ \pm}$having analogous properties: $U_{ \pm}$should be unitary intertwiners such that

$$
R_{2}^{ \pm}=U_{ \pm} \circ R_{1}^{ \pm} \circ U_{ \pm}^{-1}
$$

Such a relation presupposes that $R_{1}^{ \pm}$is unitarily equivalent to $R_{2}^{ \pm}$. If this is the case, then the maps $U_{ \pm}$are determined uniquely up to phase factors (because of Schur's lemma):

$$
U_{ \pm} \Psi^{ \pm}\left(\varphi_{1}\right)={ }_{F} e^{i \lambda_{ \pm}} \Psi^{ \pm}\left(\varphi_{2}\right)
$$

where $\lambda_{ \pm}$are arbitrary real numbers from the interval $[0,2 \pi), \Psi^{ \pm}\left(\varphi_{1}\right)$ is an arbitrary element of $\mathcal{H}_{1}^{ \pm}$and the equality ' $={ }_{F}$ ' is to be understood as follows: the symbols $\Psi^{ \pm}$on both sides denote the same mapping $S^{1} \rightarrow C$.

We are going to paste the Hilbert spaces $\mathcal{H}_{1}$ and $\mathcal{H}_{2}$ by means of $U_{ \pm}$in analogy to pasting the symplectic manifolds $\Gamma_{1}$ and $\Gamma_{2}$ by means of $\rho_{ \pm}$. Let us first give a general definition and properties.

Definition: Let $\mathcal{H}_{1}$ and $\mathcal{H}_{2}$ be two Hilbert spaces with orthogonal direct sum decompositions

$$
\begin{aligned}
& \mathcal{H}_{1}=\mathcal{H}_{1}^{0} \oplus \mathcal{H}_{1}^{\prime}, \\
& \mathcal{H}_{2}=\mathcal{H}_{2}^{0} \oplus \mathcal{H}_{2}^{\prime} .
\end{aligned}
$$

Let $U: \mathcal{H}_{1}^{0} \rightarrow \mathcal{H}_{2}^{0}$ be a unitary map. Let $\mathcal{H}$ be a Hilbert space defined by

$$
\mathcal{H}=\mathcal{H}_{1}^{0} \oplus \mathcal{H}_{1}^{\prime} \oplus \mathcal{H}_{2}^{\prime},
$$

and let two unitary maps $U_{1}: \mathcal{H}_{1} \rightarrow \mathcal{H}, U_{2}: \mathcal{H}_{2} \rightarrow \mathcal{H}$ be defined by

$$
\begin{aligned}
& U_{1}\left(\Psi_{1}^{0} ; \Psi_{1}^{\prime}\right)=\left(\Psi_{1}^{0} ; \Psi_{1}^{\prime} ; 0\right), \\
& U_{2}\left(\Psi_{2}^{0} ; \Psi_{2}^{\prime}\right)=\left(U^{-1} \Psi_{2}^{0} ; 0 ; \Psi_{2}^{\prime}\right),
\end{aligned}
$$


where $\Psi_{1}^{0} \in \mathcal{H}_{1}^{0}, \Psi_{2}^{0} \in \mathcal{H}_{2}^{0}, \Psi_{1}^{\prime} \in \mathcal{H}_{1}^{\prime}, \Psi_{2}^{\prime} \in \mathcal{H}_{2}^{\prime}$, and $(. ; . ;$.$) denotes an element of the$ orthogonal direct sum. Then the set $\left(\mathcal{H}_{1}, \mathcal{H}_{2}, U_{1}, U_{2}, \mathcal{H}\right)$ is called pasting of $\mathcal{H}_{1}$ and $\mathcal{H}_{2}$ by means of $U$.

Lemma: Let $V_{1}: \mathcal{H}_{1}^{0} \rightarrow \mathcal{H}_{1}^{0}$ and $V_{2}: \mathcal{H}_{2}^{0} \rightarrow \mathcal{H}_{2}^{0}$ be two maps satisfying the condition

$$
V_{2} \circ U=U \circ V_{1}
$$

Then

$$
U_{1} \circ V_{1} \circ U_{1}^{-1}=U_{2} \circ V_{2} \circ U_{2}^{-1} .
$$

Proof. As the domain of $V_{1}$ is $\mathcal{H}_{1}^{0}$, the domain of $U_{1} \circ V_{1} \circ U_{1}^{-1}$ is

$$
U_{1} \mathcal{H}_{1}^{0}=\left\{\left(\Psi_{1}^{0} ; 0 ; 0\right) \mid \Psi_{1}^{0} \in \mathcal{H}_{1}^{0}\right\}
$$

and we have

$$
\left(U_{1} \circ V_{1} \circ U_{1}^{-1}\right)\left(\Psi_{1}^{0} ; 0 ; 0\right)=\left(V_{1} \Psi_{1}^{0} ; 0 ; 0\right) .
$$

Similarly, the domain of $U_{2} \circ V_{2} \circ U_{2}^{-1}$ is

$$
U_{2} \mathcal{H}_{2}^{0}=\left\{\left(U^{-1} \Psi_{2}^{0} ; 0 ; 0\right) \mid \Psi_{2}^{0} \in \mathcal{H}_{2}^{0}\right\}
$$

and

$$
\left(U_{2} \circ V_{2} \circ U_{2}^{-1}\right)\left(U^{-1} \Psi_{2}^{0} ; 0 ; 0\right)=\left(U^{-1} V_{2} \Psi_{2}^{0} ; 0 ; 0\right) .
$$

Thus, if $U^{-1} \Psi_{2}^{0}=\Psi_{1}^{0}$, then

$$
\left(U_{2} \circ V_{2} \circ U_{2}^{-1}\right)\left(U^{-1} \Psi_{2}^{0} ; 0 ; 0\right)=\left(U^{-1} V_{2} U \Psi_{1}^{0} ; 0 ; 0\right)=\left(V_{1} \Psi_{1}^{0} ; 0 ; 0\right) .
$$

Q.E.D.

Returning to our system, we can set

$$
\begin{aligned}
\mathcal{H}_{1}^{0} & =\mathcal{H}_{1}=\mathcal{H}_{1}^{+} \oplus \mathcal{H}_{1}^{-}, \\
\mathcal{H}_{2}^{0} & =\mathcal{H}_{2}=\mathcal{H}_{2}^{+} \oplus \mathcal{H}_{2}^{-}, \\
U\left(\Psi_{1}^{+} ; \Psi_{1}^{-}\right) & =\left(U_{+} \Psi_{1}^{+} ; U_{-} \Psi_{1}^{-}\right), \\
\mathcal{H}^{+} & =\mathcal{H}_{1}^{+}, \mathcal{H}^{-}=\mathcal{H}_{1}^{-}, \\
\mathcal{H} & =\mathcal{H}^{+} \oplus \mathcal{H}^{-}, \\
U_{1} & =\mathrm{id}, \quad U_{2}=U^{-1} .
\end{aligned}
$$

Observe that this is a very special case of the definition above. For $V_{1}=R_{1}(\Lambda)$, $V_{2}=R_{2}(\Lambda)$, where $\Lambda \in S L(2, \mathbb{R})$, the condition (53) is satisfied for any $\lambda_{+}$and $\lambda_{-}$, because of (52).

Consider next $\hat{\sigma}_{1}$ and $\hat{\sigma}_{2}$. Let $\left\{\Psi_{m}\left(\varphi_{i}\right) \mid m \in Z_{+}\right\}$be a basis of $\mathcal{H}_{i}^{+}$, and $\left\{\Psi_{m}\left(\varphi_{i}\right) \mid m \in\right.$ $\left.Z_{-}\right\}$be a basis of $\mathcal{H}_{i}^{-}$. We obtain then from (6) that

$$
U_{ \pm} \Psi_{ \pm m}\left(\varphi_{1}\right)={ }_{F} e^{i \lambda_{ \pm}} \Psi_{ \pm m}\left(\varphi_{2}\right) \quad \forall m>0 .
$$


Hence, for $m>0$, and $\Psi_{m}\left(\varphi_{1}\right) \in \mathcal{H}_{1}^{+}$:

$$
\begin{aligned}
& U\left(\hat{\sigma}_{1}\left(\Psi_{m}\left(\varphi_{1}\right)\right)\right)=U\left(-\Psi_{-m}\left(\varphi_{1}\right)\right)={ }_{F}-e^{i \lambda_{-}} \Psi_{-m}\left(\varphi_{2}\right), \\
& \hat{\sigma}_{2}\left(U\left(\Psi_{m}\left(\varphi_{1}\right)\right)\right)={ }_{F} \quad \hat{\sigma}_{2}\left(e^{i \lambda_{+}} \Psi_{m}\left(\varphi_{2}\right)\right)=e^{i \lambda_{+}} \Psi_{-m}\left(\varphi_{2}\right),
\end{aligned}
$$

and similarly for $m>0$ and $\Psi_{-m}\left(\varphi_{1}\right) \in \mathcal{H}_{1}^{-}$. The condition (53) is then equivalent to

$$
e^{i \lambda_{+}}=-e^{i \lambda_{-}}=e^{i \lambda}
$$

Thus, the relative phase is determined uniquely from the condition

$$
R_{2}(o) \circ U=U \circ R_{1}(o)
$$

for any $o$ which maps at least one element of $\mathcal{H}_{1}^{+}$to $\mathcal{H}_{1}^{-}$.

The overall phase factor $e^{i \lambda}$ does not possess any measurable meaning. Indeed, the map $U_{2}$ in which it appears is used to carry quantum mechanical structures (the inner product and the operators) from $\mathcal{H}_{1}$ over to $\mathcal{H}$. The result of this transfer is, however, independent of $\lambda$. Let us choose a fixed $U_{2}$; all other possibilities are given by $e^{i \lambda} U_{2}$ for $\lambda \in[0,2 \pi)$. Then, two vectors $\Psi_{2}$ and $\Psi_{2}^{\prime}$ of $\mathcal{H}_{2}$ will be mapped to

$$
\Psi=e^{i \lambda} U_{2} \Psi_{2}, \quad \Psi^{\prime}=e^{i \lambda} U_{2} \Psi_{2}^{\prime},
$$

with

$$
\left(\Psi, \Psi^{\prime}\right)=\left(e^{-i \lambda} U_{2}^{-1} \Psi, e^{-i \lambda} U_{2}^{-1} \Psi^{\prime}\right)_{2}=\left(U_{2}^{-1} \Psi, U_{2}^{-1} \Psi^{\prime}\right)_{2} .
$$

Similarly, if $\hat{O}_{2}$ is an operator in $\mathcal{H}_{2}$, then its version $\hat{O}$ in $\mathcal{H}$ is defined by

$$
\hat{O}=e^{i \lambda} U_{2} \hat{O}_{2} e^{-i \lambda} U_{2}^{-1}=U_{2} \hat{O}_{2} U_{2}^{-1} \text {. }
$$

Let us, therefore, set $\lambda=0$ in eq. (57). Then the operator $U_{2}$ can be expressed in a useful simple way. Let $\Pi_{2}^{+}$and $\Pi_{2}^{-}$be the projection operators from $\mathcal{H}$ onto $\mathcal{H}_{2}^{+}$ and $\mathcal{H}_{2}^{-}$, respectively, and similarly $\Pi^{+}$and $\Pi^{-}$those from $\mathcal{H}$ onto $\mathcal{H}^{+}$and $\mathcal{H}^{-}$. Let further $I: \mathcal{H}_{2} \rightarrow \mathcal{H}$ be defined by

$$
I \Psi\left(\varphi_{2}\right)={ }_{F} \Psi(\varphi)
$$

Then we have

$$
\begin{array}{r}
U_{2}=I\left(\Pi_{2}^{+}-\Pi_{2}^{-}\right), \\
U_{2}^{-1}=I^{-1}\left(\Pi^{+}-\Pi^{-}\right),
\end{array}
$$

because of the relations (56), (57) and (54), (55) (in which $\lambda=0$ ). To check that the right hand side of (59) is inverse to that of (58), one has to use the relation

$$
I \Pi_{2}^{ \pm} I^{-1}=\Pi^{ \pm}
$$

Note that the formulae (58), (59) enable us to transfer operators and vectors from $\mathcal{H}_{2}$ to $\mathcal{H}$ immediately. 


\section{Comparison with the Dirac quantization method}

In this section we quantize our system using the straightforward Dirac quantization method and compare the results with those obtained in the preceeding section by reduction method.

Recall that the constraint of our system is

$$
C=\frac{1}{2}\left(p_{1}^{2}-q_{1}^{2}-p_{2}^{2}+q_{2}^{2}+\kappa\right)=0
$$

with $\kappa=0$. It is convenient to leave $\kappa$ arbitrary. We make the canonical transformation

$$
\tilde{q}_{1}=p_{1}, \quad \tilde{p}_{1}=-q_{1}
$$

and use the Schrödinger representation with respect to the variables $\tilde{q}_{1}$ and $q_{2}$. Thus, the quantum constraint reads

$$
\left(-\frac{\partial^{2}}{\partial \tilde{q}_{1}^{2}}-\frac{\partial^{2}}{\partial q_{2}^{2}}-\tilde{q}_{1}^{2}-q_{2}^{2}\right) \Psi=\kappa \Psi .
$$

The Casimir invariant $\hat{q}=\hat{L}_{0}^{2}-\hat{L}_{1}^{2}-\hat{L}_{2}^{2}$ can be computed using the canonical commutation relations and the constraint (60) as

$$
\hat{q} \Psi=-\left(\frac{1}{4}+\frac{\kappa^{2}}{16}\right) \Psi .
$$

Thus, the value of the Casimir invariant of $S L(2, \mathbb{R})$ for $\kappa=0$ agrees with that found in sec. 5.1.

We rewrite (61) using the polar coordinates

$$
\tilde{q}_{1}=r \cos \phi, \quad q_{2}=r \sin \phi
$$

as

$$
\left\{-\left[\frac{\partial^{2}}{\partial r^{2}}+\frac{1}{r} \frac{\partial}{\partial r}+\frac{1}{r^{2}} \frac{\partial^{2}}{\partial \phi^{2}}\right]-r^{2}\right\} \Psi=\kappa \Psi .
$$

Note that the angular momentum operator $\hat{L}_{0}$ is given by

$$
\begin{aligned}
\hat{L}_{0} & =\frac{1}{2}\left(\tilde{q}_{1} p_{2}-q_{2} \tilde{p}_{1}\right) \\
& =-\frac{i}{2} \frac{\partial}{\partial \phi} .
\end{aligned}
$$

We choose the wavefunctions to be eigenfunctions of $\hat{L}_{0}$ :

$$
\hat{L}_{0} \Psi_{m}^{\kappa}(r, \phi)=\frac{m}{2} \phi, \quad \Psi_{m}^{\kappa}(r, \phi)=\psi_{m}^{\kappa}(r) e^{i m \phi} .
$$


The radial wave equation for $\psi_{m}^{\kappa}(r)$ is

$$
\left[\frac{d^{2}}{d r^{2}}+\frac{1}{r} \frac{d}{d r}-\frac{m^{2}}{r^{2}}+r^{2}+\kappa\right] \psi_{m}^{\kappa}(r)=0 .
$$

The solution that is regular at $r=0$ is given by

$$
\psi_{m}^{\kappa}(r) \propto \frac{1}{r} M_{-\frac{i}{4} \kappa, \frac{|m|}{2}}\left(i r^{2}\right)
$$

where

$$
M_{\lambda, \mu}(y)=y^{\mu+\frac{1}{2}} e^{-\frac{y}{2}} \Phi\left(\mu-\lambda+\frac{1}{2}, 2 \mu+1 ; y\right)
$$

is the Whittaker function and

$$
\Phi(\alpha, \gamma ; y)=1+\frac{\alpha}{\gamma} \frac{y}{1 !}+\frac{\alpha(\alpha+1)}{\gamma(\gamma+1)} \frac{y^{2}}{2 !}+\cdots
$$

is the confluent hypergeometric function (see [13], p. 1059, 9.220.1-3). To find the normalization factor we need to evaluate

$$
\lim _{L \rightarrow \infty} \int_{0}^{L} r d r d \phi \overline{G_{m}^{\kappa}(r, \phi)} G_{m^{\prime}}^{\kappa^{\prime}}(r, \phi)
$$

where

$$
G_{m}^{\kappa}(r, \phi):=\frac{1}{r} M_{-\frac{i}{4} \kappa, \frac{|m|}{2}}\left(i r^{2}\right) e^{i m \phi} .
$$

By multiplying by $\kappa-\kappa^{\prime}$ and using (66), one can rewrite the integral (67) as a "surface term" at $r=L$. Then, it can be computed using the large $r$ behavior of

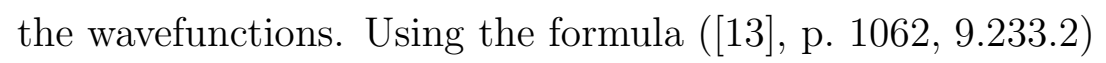

$$
\begin{aligned}
M_{\lambda, \mu}(y)= & \frac{\Gamma(2 \mu+1)}{\Gamma\left(\mu-\lambda+\frac{1}{2}\right)} e^{-i \pi \lambda} W_{-\lambda, \mu}\left(e^{-i \pi} y\right) \\
& +\frac{\Gamma(2 \mu+1)}{\Gamma\left(\mu+\lambda+\frac{1}{2}\right)} \exp \left[-i \pi\left(\lambda-\mu-\frac{1}{2}\right)\right] W_{\lambda, \mu}(y)
\end{aligned}
$$

and the fact that the Whittaker function behaves as $W_{\lambda, \mu}(y) \sim e^{-\frac{y}{2}} y^{\lambda}$ for large $|y|$ with $|\arg y|<\pi([13]$, p. 1061, 9.227), we find for large $r$

$$
\frac{1}{r} M_{-\frac{i}{4} \kappa, \frac{m}{2}}\left(i r^{2}\right) \sim \frac{m ! e^{-\frac{\pi \kappa}{8}}}{\Gamma\left(\frac{m+1}{2}+\frac{i \kappa}{4}\right)} e^{i r^{2}} r^{i \frac{\kappa}{2}-1}+\frac{m ! e^{\frac{m+1}{2} \pi i} e^{-\frac{\pi \kappa}{8}}}{\Gamma\left(\frac{m+1}{2}-\frac{i \kappa}{4}\right)} e^{-i r^{2}} r^{-i \frac{\kappa}{2}-1}
$$

By using this equation, dropping rapidly oscillating functions of $\kappa$ and $\kappa^{\prime}$ and using

$$
\lim _{\alpha \rightarrow+\infty} \frac{\sin \alpha x}{x}=\pi \delta(x)
$$


we find

$$
\lim _{L \rightarrow \infty} \int_{0}^{L} r d r d \phi \overline{G_{m}^{\kappa}(r, \phi)} G_{m^{\prime}}^{\kappa^{\prime}}(r, \phi)=\frac{8 \pi^{2}(|m| !)^{2} e^{-\frac{\pi \kappa}{4}}}{\left|\Gamma\left(\frac{|m|+1}{2}+\frac{i}{4} \kappa\right)\right|^{2}} \delta_{m m^{\prime}} \delta\left(\kappa-\kappa^{\prime}\right) .
$$

It is clear that this integral is divergent if we let $\kappa=\kappa^{\prime}=0$. This divergence simply represents the volume of the "gauge group" generated by the constraint. This implies that the inner product of wavefunctions satisfying the constraint should be defined by dropping $2 \pi \delta(0)$, as recently proposed by Marolf 14]. Hence we define

$$
\Psi_{m}^{\kappa}(r, \phi):=\frac{\Gamma\left(\frac{|m|+1}{2}+\frac{i}{4} \kappa\right)}{\sqrt{4 \pi}|m| !} \times \frac{1}{r} M_{-\frac{i}{4} \kappa, \frac{|m|}{2}}\left(i r^{2}\right) e^{i m \phi} .
$$

They satisfy

$$
\int r d r d \phi \overline{\Psi_{m}^{\kappa}(r, \phi)} \Psi_{m^{\prime}}^{\kappa^{\prime}}(r, \phi)=2 \pi e^{-\frac{\pi \kappa}{4}} \delta\left(\kappa-\kappa^{\prime}\right) \delta_{m m^{\prime}}
$$

Then the normalized basis functions satisfying the constraint (61) with $\kappa=0$ are given by

$$
\Psi_{m}(r, \phi)=\Psi_{m}^{0}(r, \phi)
$$

The inner product is defined simply by

$$
\left\langle\Psi_{m} \mid \Psi_{m^{\prime}}\right\rangle=\delta_{m m^{\prime}}
$$

Now, notice that $r^{m} e^{i m \phi}=\left(\tilde{q}_{1}+i q_{2}\right)^{m}$. By defining

$$
\begin{aligned}
z & :=\frac{\tilde{q}_{1}+i q_{2}}{2}, \\
\bar{z} & :=\frac{\tilde{q}_{1}-i q_{2}}{2},
\end{aligned}
$$

and using the expression (69) for $\Psi_{m}^{\kappa}(r, \phi)$ we find

$$
\Psi_{m}^{\kappa}=\frac{i^{\frac{m+1}{2}} e^{-2 i \bar{z} z}}{\sqrt{4 \pi}} \sum_{k=0}^{\infty} \frac{\Gamma\left(\frac{m+1}{2}+k+\frac{i \kappa}{4}\right)}{(m+k) ! k !} i^{k}(2 \bar{z})^{k}(2 z)^{k+m} \quad(m \geq 0)
$$

and

$$
\Psi_{m}^{\kappa}=\frac{i^{\frac{|m|+1}{2}} e^{-2 i \bar{z} z}}{\sqrt{4 \pi}} \sum_{k=0}^{\infty} \frac{\Gamma\left(\frac{|m|+1}{2}+k+\frac{i \kappa}{4}\right)}{(|m|+k) ! k !} i^{k}(2 z)^{k}(2 \bar{z})^{k+|m|} \quad(m<0) .
$$

\footnotetext{
${ }^{5}$ This procedure is closely related to that introduced to deal with "linearization instabilities"
} 15. 
It is straightforward to derive the following formulae:

$$
\begin{aligned}
& \left(\frac{1}{2} \frac{\partial}{\partial z}+i \bar{z}\right) \Psi_{m}^{\kappa}=i^{\frac{1}{2}} \Psi_{m-1}^{\kappa-2 i}, \\
& \left(\frac{1}{2} \frac{\partial}{\partial \bar{z}}+i z\right) \Psi_{m}^{\kappa}=i^{\frac{1}{2}} \Psi_{m+1}^{\kappa-2 i}, \\
& \left(\frac{1}{2} \frac{\partial}{\partial z}-i \bar{z}\right) \Psi_{m}^{\kappa}=\left(\frac{m-1}{2}+\frac{i}{4} \kappa\right) i^{\frac{1}{2}} \Psi_{m-1}^{\kappa+2 i}, \\
& \left(\frac{1}{2} \frac{\partial}{\partial \bar{z}}+i z\right) \Psi_{m}^{\kappa}=\left(-\frac{m+1}{2}+\frac{i}{4} \kappa\right) i^{\frac{1}{2}} \Psi_{m+1}^{\kappa+2 i} .
\end{aligned}
$$

Noting that

$$
\begin{aligned}
\tilde{q}_{1} & =z+\bar{z} \\
q_{2} & =i(\bar{z}-z) \\
\tilde{p}_{1} & =-\frac{i}{2}\left(\frac{\partial}{\partial z}+\frac{\partial}{\partial \bar{z}}\right) \\
p_{2} & =\frac{1}{2}\left(\frac{\partial}{\partial z}-\frac{\partial}{\partial \bar{z}}\right)
\end{aligned}
$$

we find

$$
\begin{aligned}
& \hat{L}_{+}=-i\left(\frac{1}{4} \frac{\partial^{2}}{\partial \bar{z}^{2}}+z^{2}\right), \\
& \hat{L}_{-}=i\left(\frac{1}{4} \frac{\partial^{2}}{\partial z^{2}}+\bar{z}^{2}\right),
\end{aligned}
$$

where $\hat{L}_{ \pm}$are defined by (49). It is easy to find how these operators act on $\Psi_{m}^{\kappa}$ using (72)-(75):

$$
\begin{aligned}
& \hat{L}_{+} \Psi_{m}^{\kappa}=\left(-\frac{m+1}{2}+\frac{i \kappa}{4}\right) \Psi_{m+2}^{\kappa}, \\
& \hat{L}_{-} \Psi_{m}^{\kappa}=\left(-\frac{m-1}{2}-\frac{i \kappa}{4}\right) \Psi_{m-2}^{\kappa},
\end{aligned}
$$

in agreement with relation (50) obtained in sec. 5.1.

Next, we consider the operators $\hat{\psi}_{1}$ and $\hat{\psi}_{2}$ whose classical counterparts are defined in sec. 2.3. By expressing the operators $\hat{Q}_{1}, \hat{Q}_{2}, \hat{P}_{1}$, and $\hat{P}_{2}$ in terms of $z, \bar{z}, \partial / \partial z$, and $\partial / \partial \bar{z}$, one finds

$$
\begin{aligned}
e^{i \hat{\psi}_{1}} & =\left(\frac{1}{2} \frac{\partial}{\partial \bar{z}}+i z\right)\left(\frac{1}{2} \frac{\partial}{\partial z}+i \bar{z}\right)^{-1} \\
e^{i \hat{\psi}_{2}} & =-\left(\frac{1}{2} \frac{\partial}{\partial \bar{z}}-i z\right)\left(\frac{1}{2} \frac{\partial}{\partial z}-i \bar{z}\right)^{-1}
\end{aligned}
$$


Notice that there is no factor ordering ambiguity. However, these operators are illdefined for $\kappa=0$. For this reason we regularize them by allowing $\kappa$ to be nonzero, and then take the limit $\kappa \rightarrow 0$. We find using (72)-(75)

$$
\begin{aligned}
e^{i \hat{\psi}_{1}} \Psi_{m}^{\kappa} & =\Psi_{m+2}^{\kappa}, \\
e^{i \hat{\psi}_{2}} \Psi_{m}^{\kappa} & =\frac{m+1-\frac{i}{2} \kappa}{m+1+\frac{i}{2} \kappa} \Psi_{m+2}^{\kappa},
\end{aligned}
$$

By taking the limit $\kappa \rightarrow 0$, we have

$$
e^{i \hat{\psi}_{1}} \Psi_{m}=\Psi_{m+2} \quad(\text { for all } m) .
$$

and

$$
\begin{aligned}
e^{i \hat{\psi}_{2}} \Psi_{m} & =\Psi_{m+2} \quad(m \neq-1), \\
e^{i \hat{\psi}_{2}} \Psi_{-1} & =-\Psi_{+1},
\end{aligned}
$$

In sec. 5, quantization was performed in the representations in which $\hat{\psi}_{1}$ or $\hat{\psi}_{2}$ is diagonalized. The wavefunction in our Schrödinger representation,

$$
\Phi(r, \phi)=\sum_{m=-\infty}^{+\infty} c_{m} \Psi_{m}(r, \phi),
$$

is written in those representations as

$$
\begin{aligned}
\Psi^{(1)}\left(\varphi_{1}\right) & =\sum_{m=-\infty}^{+\infty} c_{m} e^{i \frac{m}{2} \varphi_{1}} \\
\Psi^{(2)}\left(\varphi_{2}\right) & =\sum_{m=+1}^{+\infty} c_{m} e^{i \frac{m}{2} \varphi_{2}}-\sum_{m=-\infty}^{-1} c_{m} e^{i \frac{m}{2} \varphi_{2}},
\end{aligned}
$$

where the summation is over odd $m$. We have identified $\psi_{\lambda}$ with $\varphi_{\lambda}(\lambda=1,2)$ since the variable $e^{i \psi_{\lambda}}$ is naturally associated with $\Gamma_{\lambda}$ [see Sec. 4, below (39))]. The maps $\Phi \rightarrow \Psi^{(i)}$ are unitary with the inner products of $\Psi^{(i)}$ defined as in (40). These wavefunctions indeed satisfy

$$
\begin{aligned}
e^{i \hat{\psi}_{i}} \Psi\left(\varphi_{i}\right) & =e^{i \varphi_{i}} \Psi^{(i)}\left(\varphi_{i}\right), \\
\hat{L}_{0} \Psi^{(i)}\left(\varphi_{i}\right) & =-i \frac{\partial}{\partial \varphi_{i}} \Psi^{(i)}\left(\varphi_{i}\right), \\
\hat{L}_{ \pm} \Psi^{(i)}\left(\varphi_{i}\right) & =i e^{ \pm i \frac{\varphi_{i}}{2}} \frac{\partial}{\partial \varphi_{i}} e^{ \pm i \frac{\varphi_{i}}{2}} \Psi^{(i)}\left(\varphi_{i}\right) .
\end{aligned}
$$

Finally, we consider the discrete operator $\hat{\sigma}$ corresponding to the classical transformation defined in sec. 3.2. The operator $\hat{\sigma}$ multiplies $\tilde{q}_{1}$ and $\tilde{p}_{1}=-i \partial / \partial \tilde{q}_{1}$ by -1 in the Dirac quantization adopted here. Hence,

$$
\hat{\sigma} z=-\bar{z}, \quad \hat{\sigma} \bar{z}=-z .
$$


Using (70) and (71) we find

$$
\hat{\sigma} \Psi_{m}=-\Psi_{-m} .
$$

(Recall that we have restricted $m$ to be odd.) Thus, from (77) and (76) the corresponding operator $\hat{\sigma}$ on $\Psi^{(1)}$ and $\Psi^{(2)}$ can be found as

$$
\begin{aligned}
\hat{\sigma} \Psi^{(1)}\left(\varphi_{1}\right) & =\Psi^{(1)}\left(2 \pi-\varphi_{1}\right), & \varphi \in[0,2 \pi), \\
& =\Psi^{(1)}\left(6 \pi-\varphi_{1}\right), & \varphi_{1} \in[2 \pi, 4 \pi) \\
\hat{\sigma} \Psi^{(2)}\left(\varphi_{2}\right) & =\Psi^{(2)}\left(4 \pi-\varphi_{2}\right), & \varphi_{2} \in[0,4 \pi) .
\end{aligned}
$$

It is interesting to note that these formulae coincide with eqs. (46) - (48).

\section{Time levels}

Consider the transversal surface $\Gamma_{i}$. The subgroup of $O_{2}^{+}(2,1)$ that leaves $\Gamma_{i}$ invariant is generated by $\Lambda_{0}(t), t \in \mathbb{R}$, and $\sigma$. All other generators of $\mathrm{SO}_{2}(2,1)$ are, therefore, possible candidates for Hamiltonians.

The formulae (22), (23), (29), (30) and (31) show how the subgroup $\Lambda_{\theta}(t)$ shifts the surface $\Gamma_{i}$. Let us denote $\Gamma_{i}(t, \theta)$ the result; then, $\Gamma_{i}(t, \theta)$ is a two-dimensional family of time levels. We will demonstrate that this is a complete family.

The intersection of $\Gamma_{i}(t, \theta)$ with the surface $\psi=$ const is a curve

$$
x=T N_{\theta}(\psi, t)
$$

for $i=1$ and

$$
y=T N_{\theta}(\psi, t)
$$

for $i=2$. If we fix $\theta$ and $\psi$, then the function $N_{\theta}(\psi, t)$ has a minimum at $t_{m}(\theta, \psi)$,

$$
t_{m}(\theta, \psi)=\operatorname{arctanh} \sin (\psi+\theta)
$$

given by

$$
N_{\theta}\left(\psi, t_{m}(\theta, \psi)\right)=\sqrt{|\cos (\psi+\theta)|} .
$$

The minimum is positive unless $\psi \in\left\{\frac{\pi}{2}-\theta, \frac{3 \pi}{2}-\theta, \frac{5 \pi}{2}-\theta, \frac{7 \pi}{2}-\theta\right\}$, where $t_{m}(\theta, \psi)=$ $\pm \infty$ and $N_{\theta}\left(\psi, t_{m}(\theta, \psi)\right)=0$. Thus, the surfaces $\Gamma_{i}(t, \theta)$ for a fixed $\theta$ do not sweep the whole domain of $\Gamma_{i}$ but only a part satisfying

$$
x \geq T \sqrt{|\cos (\psi+\theta)|}
$$

for $i=1$ and

$$
y \geq T \sqrt{|\cos (\psi+\theta)|}
$$


for $i=2$. Hence, each $L_{\theta}$ can only be made a partial Hamiltonian. However, the set of $\Gamma_{i}(t, \theta)$ for all $t$ and $\theta$ cover the whole $\mathrm{D}\left(\Gamma_{i}\right)$. Indeed,

$$
\begin{aligned}
& \mathrm{D}\left(\Gamma_{1}\right)=\{(x, y, \psi) \in \tilde{\Gamma} \mid x>0,-\infty<y<\infty, 0<\psi \leq 4 \pi\} \\
& \mathrm{D}\left(\Gamma_{2}\right)=\{(x, y, \psi) \in \tilde{\Gamma} \mid y>0,-\infty<x<\infty, 0<\psi \leq 4 \pi\} .
\end{aligned}
$$

Thus, the one-dimensional family of generators $L_{\theta}, \theta \in(0,2 \pi)$, yields a complete system of partial Hamiltonians for each of the surfaces $\Gamma_{1}$ and $\Gamma_{2}$. We obtain two disjoint systems of time levels (no element of our first-class canonical group maps a time level of one system into a time level of the second system). Let us limit ourselves to $\Gamma_{1}(t, \theta)$, and consider the problem of the "same measurement" at different time level. (We leave out the index "1" referring to the surface $\Gamma_{1}$ in the rest of this section. For example, $\varphi_{1}$ is denoted simply by $\varphi$.)

According to Theorem 9 in I, we can calculate the change in the result of the "same measurement" represented by a perennial $o$ between two neighbouring levels $\Gamma_{1}(t, \theta)$ and $\Gamma_{1}(t+d t, \theta)$ as follows. We restrict $o$ to $\Gamma_{1}$ obtaining the time-independent observable $\tilde{o}$ and calculate its value along the "physical trajectory" generated in $\Gamma_{1}$ by the "Hamiltonian" $-\left.L_{\theta}\right|_{\Gamma_{1}}$. This is the Schrödinger picture. To be more concrete, we have the observables given by eqs. (38) and (39) with $i=1$, and we have the Hamiltonians

$$
H_{\theta}=\left.L_{\theta}\right|_{\Gamma_{1}}=-L_{1} \cos \theta-L_{2} \sin \theta=l \cos (\varphi+\theta)
$$

In the quantum theory, the observables are represented by the time-independent operators $\hat{L}_{0}, \hat{L}_{1}, \hat{L}_{2}$ and $\hat{\sigma}$ given by eqs. (41)-(43) and (46)-(48), whereas the Hamiltonian operator $\hat{H}_{\theta}$ can be calculated with the result

$$
\hat{H}_{\theta}=-i \cos (\varphi+\theta) \frac{\partial}{\partial \varphi}+\frac{i}{2} \sin (\varphi+\theta) .
$$

This is, of course, $-\hat{L}_{\theta}$ as given by eq. (45). The corresponding Schrödinger equation

$$
i \frac{\partial \Psi}{\partial t}=\hat{H}_{\theta} \Psi
$$

is then easily integrated, if we use eqs. (44) and (45), with the result

$$
\Psi(\theta, t, \varphi)=\left(U\left(\Lambda_{\theta}(t)\right) \Psi\right)(\varphi)=\frac{1}{N_{\theta}(\varphi, t)} \Psi\left(F_{\theta}(\varphi, t)\right)
$$

Thus, we have a four-dimensional family of unitary operators, $U^{-1}\left(\Lambda_{\theta^{\prime}}\left(t^{\prime}\right)\right) U\left(\Lambda_{\theta}(t)\right)$, that bring the wave function $\Psi\left(\theta^{\prime}, t^{\prime}, \varphi\right)$ at the time level $\Gamma_{1}\left(\theta^{\prime}, t^{\prime}\right)$ to the wave function $\Psi(\theta, t, \varphi)$ at the time level $\Gamma_{1}(\theta, t)$. The corresponding Heisenberg picture is easy to obtain, and we leave it to the reader as an exercise. 
An important observation is the following. For the system we are studying in the present paper, the full Hilbert space $\mathcal{H}$ is identical to the Hilbert space $\mathcal{H}_{1}$ corresponding to the reduced subsystem $\left(\Gamma_{1}, \Omega_{1}\right)$. Hence, our operators that represent both observables and Hamiltonians are applicable to all vectors of $\mathcal{H}$. Hence, we have already a complete quantum theory and a complete "truncated" many-fingered time evolution. Clearly, the set of classical orbits of measure zero that is missing in $\left(\Gamma_{1}, \Omega_{1}\right)$ (namely orbits with $x=0$ ) does not play any role in the quantum theory. If one would like to study wave packets concentrated along these orbits, then one clearly must use the same Hilbert space $\mathcal{H}$, but now "in the form" of $\mathcal{H}_{2}$ (which is unitarily equivalent).

To summarize: we have found a complete two-dimensional family of time levels at the constraint hypersurface of the system and we have constructed the fourdimensional family of unitary operators representing the corresponding ("truncated many-fingered") time evolution.

\section{Acknowledgements}

We are grateful for discussions with J. Louko, D. Marolf, J. Mourão, and R. Tate. P.H. and A.H. acknowledges the support of the Swiss Nationalfonds. J.T. is indebted for the warm hospitality of the Institute for Theoretical Physics in Bern. He also acknowledges the support of the Tomalla-Stiftung Fund and of the Grant Agency of Czech Republic.

\section{References}

[1] P. Hájíček, Group quantization of parametrized systems I. Time levels. Preprint BUTP-94/21.

[2] P.A.M. Dirac, Rev. Mod. Phys. 21 (1949) 392.

[3] A. Ashtekar, Lectures on Non-Perturbative Canonical Gravity. World Science, Singapore, 1991;

A. Ashtekar, R.S. Tate, C. Uggla, Int. J. Mod. Phys. D2, (1993) 15;

A. Ashtekar, R.S. Tate, C. Uggla, Minisuperspaces: Symmetries and Quantization. Preprint SU-CP-92/2-5 (1993);

A. Ashtekar, R.S. Tate, An Algebraic extension of Dirac Quantization: Examples. Preprint, CGPG-94/6-1, gr-qc/9405073.

[4] C.J. Isham, in Relativity, Groups and Topology II. Proceedings of the Les Houches Summer School, Les Houches, France, 1983. R. Stora and B.S. DeWitt, eds. North-Holland, Amsterdam, 1984, pp. 1061-1290; 
C.J. Isham and A.C. Kakas, Class. Quantum Gravit. 1 (1984) 621; 1 (1984) 633 ;

C.J. Isham and N. Linden, Class. Quantum Gravit. 5 (1988) 71;

H. Zainuddin, Phys Rev. D 40 (1989) 636; C. Rovelli, Nuovo Cim. 100B (1987) 343.

[5] K. V. Kuchař, Time and Interpretation of Quantum Gravity. In: Proceedings of the 4th Canadian Conference on General Relativity and Relativistic Astrophysics. World Science, Singapore, 1992).

[6] P. Hájiček, Quantization of Systems with Constraints. In: Canonical Gravity: From Classical to Quantum. Eds. J. Ehlers and H. Friedrich. Springer, Berlin, 1994.

[7] P. Hájičcek, J. Math. Phys. 30 (1989) 2488.

[8] W. Greub, H.-R. Petry, J. Math. Phys. 16, (1975) 1347.

[9] H.D. Doebner, P. Šťovíček, J. Tolar, Czech. J. Phys. B 31, (1981).

[10] V. Bargmann, Ann. Math. 48 (1947) 568.

[11] A.O. Barut, C. Fronsdal, Proc. Roy. Soc. A 287 (1965) 532.

[12] Y. Ohnuki, Unitary Representations of the Poincaré Group and Relativistic Wave Equations, World Scientific, Singapore 1988.

[13] I.S. Gradshteyn and I.M. Ryzhik, Tables of Integrals, Series, and Products, edited by A. Jeffrey (Academic, New York, 1980).

[14] D. Marolf, Quantum Observables and Recollapsing Dynamics, gr-qc/9404053.

[15] A. Higuchi, Class. Quantum Grav. 8 (1991) 1983. 